\title{
First-principles calculation of atomic structure, stability and electronic structure of TaB2/SiC interface
}

\section{Gong Cheng ( $\sim$ gcheng@cqu.edu.cn )}

Lanzhou Institute of Physics China Academy of Space Technology

\section{Yuqing Xiong}

Lanzhou Institute of Physics China Academy of Space Technology

Hui Zhou

Lanzhou Institute of Physics China Academy of Space Technology

\section{Yanchun He}

Lanzhou Institute of Physics China Academy of Space Technology

\section{Kaifeng Zhang}

Lanzhou Institute of Physics China Academy of Space Technology

\section{Shengzhu Cao}

Lanzhou Institute of Physics China Academy of Space Technology

\section{Keliang Wang}

Lanzhou Institute of Physics China Academy of Space Technology

\section{Research Article}

Keywords: First-principles, TaB2/SiC interface, Work of adhesion, Interfacial stability, Electronic structure

Posted Date: December 10th, 2020

DOl: https://doi.org/10.21203/rs.3.rs-123800/v1

License: (c) (i) This work is licensed under a Creative Commons Attribution 4.0 International License. Read Full License

Version of Record: A version of this preprint was published at Modelling and Simulation in Materials Science and Engineering on April 14th, 2021. See the published version at https://doi.org/10.1088/1361651X/abe925. 


\title{
First-principles calculation of atomic structure, stability and electronic structure of $\mathrm{TaB}_{2} / \mathrm{SiC}$ interface
}

Gong Cheng, Yuqing Xiong*, Hui Zhou, Yanchun He, Kaifeng Zhang, Shengzhu Cao,

\author{
Keliang Wang
}

Science and Technology on Vacuum Technology and Physics Laboratory, Lanzhou Institute of Physics, Lanzhou 730000, China

\begin{abstract}
The atomic structure, interface stability and electronic interaction of $\mathrm{TaB}_{2}(0001) / \mathrm{SiC}(111)$ interfaces were investigated by first principles study. The study found that the termination atom and stacking position are the key factors affecting the bonding strength and stability of the interface. On the basis of considering work of

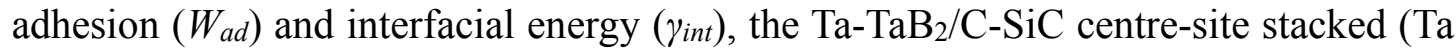
$\mathrm{CS}-\mathrm{C}$ ) and $\mathrm{B}-\mathrm{TaB}_{2} / \mathrm{C}-\mathrm{SiC}$ center-site stacked (B-CS-C) configurations were recognized as the most stable structures from ten different interface models. Electronic interaction of the two most stable interfaces were revealed by analyzing the charge density distribution, charge density difference and partial density of states (PDOS), and it was found that ionic and metallic bond coexisted in Ta CS-C interface, while covalent bond played a dominant role in B-CS-C interface.
\end{abstract}

Keywords: First-principles, $\mathrm{TaB}_{2} / \mathrm{SiC}$ interface, Work of adhesion, Interfacial stability, Electronic structure

\section{Introduction}

Carbon materials, especially carbon fiber reinforced carbon matrix $(\mathrm{C} / \mathrm{C})$ composite materials, with unique advantages of low density, low thermal expansion coefficient (CTE), high strength, high thermal conductivity and structural stability, are considered as the most promising thermal structure material and have aroused special interests in aeronautic and aerospace fields ${ }^{[1-5]}$, such as hypersonic vehicles and rocket propulsion systems. However, the application of $\mathrm{C} / \mathrm{C}$ composites facing huge challenges due to its sensitivity to the oxidants at a temperature higher than $\sim 723 \mathrm{~K}^{[6,7]}$. Considering the superiority of $\mathrm{C} / \mathrm{C}$ composites as thermal structural material, it is necessary to address the problem of easy oxidation, to extend its service life. High-temperature antioxidation protective coating was considered as an effective measure. 
With low oxygen permeability, high chemical stability, excellent self-healing ability, and a similar coefficient of thermal expansion (CTE) with $\mathrm{C} / \mathrm{C}$ substrate, SiC-based coatings have become the preferred oxidation-resistant coating material for $\mathrm{C} / \mathrm{C}$ composites ${ }^{[8-10]}$. Among the coating components, SiC combined with the ultra-high temperature ceramics (UHTCs), including $\mathrm{ZrB}_{2}, \mathrm{ZrC}, \mathrm{HfB}_{2}, \mathrm{TaB}_{2}$ etc., exhibits unparalleled oxidation resistance, good chemical and thermo-physical compatibility. Wang et al. ${ }^{[11]}$ examined the anti-oxidation performance of the $\mathrm{C} / \mathrm{C}$ composite coated with gradient $\mathrm{HfB}_{2}$ modified SiC coating at 1773, 1873 and $1973 \mathrm{~K}$. The results suggested that the $\mathrm{HfB}_{2}-\mathrm{SiC}$ coating with good crystallization exhibits excellent oxidation resistance at $1773 \mathrm{~K}$, with a mass loss of $0.17 \%$ after 800 hours, and the hafnium compound could consume the crack formation energy and block the crack propagation during the oxidation process. Ren et al. ${ }^{[12]}$ investigated the anti-oxidation ability of $\mathrm{ZrB}_{2}-\mathrm{SiC}$ coating and indicated that the excellent oxidation protective ability of the $\mathrm{ZrB}_{2}-\mathrm{SiC}$ gradient coating can be maintained for more than 207 hours at $1773 \mathrm{~K}$. Zirconium compound played a role of pinning phase, which not only improves the oxidation resistance of the coating, but also consumes the crack energy and prevents the propagation of the crack. Li et al. ${ }^{[13]}$ studied the anti-ablation properties of the $\mathrm{ZrC}$ $\mathrm{SiC}$ coating prepared by solid-phase infiltration, and proposed that the dense $\mathrm{ZrC}-\mathrm{SiC}$ coating is composed of $\mathrm{ZrC}$ and $\mathrm{SiC}$ phases. The coating and the matrix are chemically bonded and have a good bonding strength. The excellent ablation resistance of the $\mathrm{ZrC}$ $\mathrm{SiC}$ coating enhanced with the increase of $\mathrm{ZrC}$ content, this is due to the fact that zirconium compound absorbs a large amount of heat during the melting process, thereby reducing the temperature of the coating surface, in addition, the formed $\mathrm{Zr}-\mathrm{Si}$ $\mathrm{O}$ glass phase could also prevent the permeation of oxygen. Jiang et al. ${ }^{[14]}$ examined the anti-oxidation and anti-ablation performance of $\mathrm{TaB}_{2}-\mathrm{SiC}-\mathrm{Si}$ coating and proved that the $\mathrm{TaB}_{2}-\mathrm{SiC}-\mathrm{Si}$ coating could protect graphite from oxidation for 321 hours and 168 hours at $1020^{\circ} \mathrm{C}$ and $1550{ }^{\circ} \mathrm{C}$, respectively. This is mainly because the $\mathrm{Ta}_{2} \mathrm{O}_{5}-\mathrm{SiO}_{2}$ barrier layer prevents the oxidizing gas from diffusing into the coating. Researches on SiC-UHTCs coatings suggested that the excellent coating performance largely comes from the following two aspects: Ultra-high temperature ceramic bonded well with the 
$\mathrm{SiC}$ and could consume crack propagation energy and deflect the crack; Glass phase on the coating surface blocks the oxygen penetration. Obviously, the interface between UHTCs and $\mathrm{SiC}$ in UHTCs-SiC coatings has a significant impact on its performance. Therefore, it is therefore of great importance to obtaining detailed heterointerface information between UHTCs and SiC, which is beneficial for us to have a good understanding of the properties of the UHTCs-SiC coatings. Taking into account the difficulty of exploring the interface with experimental methods, a first-principles calculation based on density functional theory (DFT) has been employed to reveal the interface characteristics at atomic and electronic scales, and has been successfully applied to $\mathrm{SiC} / \mathrm{ZrB}_{2}{ }^{[15-17]} \mathrm{HfB}_{2} / \mathrm{SiC}{ }^{[18]}$, and $\mathrm{ZrC} / \mathrm{SiC}{ }^{[19]}$ systems. However, up to now, the interface characteristics between $\mathrm{TaB}_{2}$ and $\mathrm{SiC}$ in $\mathrm{TaB}_{2}-\mathrm{SiC}$ coating are still mysterious, although there are many practical applications of the $\mathrm{TaB}_{2}-\mathrm{SiC}$ coating as anti-oxidation and anti-ablation materials. Therefore, the purpose of this paper is to bring to light the interface characteristics between $\mathrm{TaB}_{2}$ and $\mathrm{SiC}$ and find the most possible interface configurations by analyzing the atomic structures, bonding strength, stability, and electronic structure within the framework of the first-principles study.

\section{Calculation methods}

In this work, a first-principles study was applied to the $\mathrm{TaB}_{2} / \mathrm{SiC}$ interface, using Cambridge Serial Total Energy Package (CASTEP) software ${ }^{[20]}$, which is based on density functional theory (DFT). The Perdew-Burke-Ernzerhof (PBE) functional of Generalized Gradient Approximation (GGA) was selected to describe the exchangecorrelation energy ${ }^{[21,22]}$. The ion-electron interactions of Ta, B, Si and C atoms were described by the plane-wave ultrasoft pseudopotential ${ }^{[23]}$. The convergence test of the kinetic energy cut-off value and K-point meshing were conducted based on the method reported in our previous study ${ }^{[18]}$, consequently, the plane wave cut-off energy for bulk $\mathrm{TaB}_{2}$ and $\mathrm{SiC}$ was set to $350 \mathrm{eV}$, and grid meshing of $9 \times 9 \times 8$ and $8 \times 8 \times 8$ was applied to $\mathrm{TaB}_{2}$ and $\mathrm{SiC}$, respectively. For the slabs and interfaces, a cut-off energy of $350 \mathrm{eV}$ and a k-point grid of $9 \times 9 \times 1$ was adopted. Periodic boundary conditions applied for slabs and interfaces, and a vacuum layer of $15 \AA$ was inserted between the above and below surfaces to minimize their interaction. 
Geometric optimization was imposed on all calculations through the BroydenFletcher-Goldfarb-Shanno (BFGS) algorithm to keep the atomic coordinates fully relaxed and reach the ground state ${ }^{[24,25]}$. Geometric optimization was considered complete when the following convergence criteria are satisfied: $5 \times 10^{-6} \mathrm{eV} /$ atom for energy, $0.01 \mathrm{eV} / \AA$ for maximum force, and $5 \times 10^{-4} \AA$ for maximum displacement.

\section{Results and discussions}

\subsection{Bulk properties}

As presented in Fig. 1 (a) and (b), $\mathrm{TaB}_{2}$ and $\mathrm{SiC}$ belong to the hexagonal and cubic system, with a symmetry group of P6/mmm (No. 191) and F-43m (No. 216), respectively. Inspecting Table 1 that our calculated lattice constant "a" and "c" of $\mathrm{TaB}_{2}$ are: $\mathrm{a}=3.102 \AA, \mathrm{c}=3.261 \AA$, which is good in line with the theoretical and experiment results ${ }^{[26-29]}$. For $\mathrm{SiC}$, the calculated lattice constant "a" of $4.361 \AA$, is also very consistent with the experimental and theoretical values in Ref. ${ }^{[15,30-32]}$. Therefore, the consistency between our calculated lattice constant and reference values also provides evidence that our calculation method and subsequent calculation results are credible.

(a)

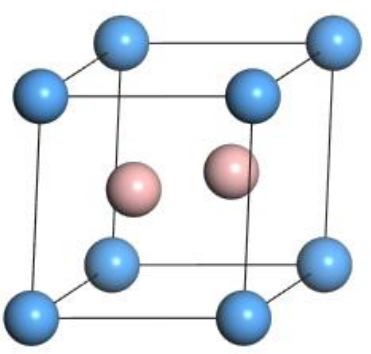

(b)

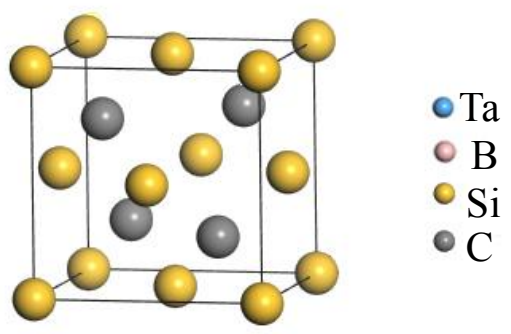

Fig. 1 The crystal structures of $\mathrm{TaB}_{2}(\mathrm{a})$ and $\mathrm{SiC}(\mathrm{b})$.

Table 1 Calculated lattice constants of bulk $\mathrm{TaB}_{2}$ and $\mathrm{SiC}$, and other experimental and theoretical calculation results.

\begin{tabular}{cccccc}
\hline & \multicolumn{3}{c}{$\mathrm{TaB}_{2}$} & & $\mathrm{SiC}$ \\
\cline { 2 - 3 } \cline { 5 - 5 } & $\mathrm{a}(\AA)$ & $\mathrm{c}(\AA)$ & & $\mathrm{a}(\AA)$ \\
\hline Present & 3.102 & 3.261 & & Present & 4.361 \\
Calc. ${ }^{[26]}$ & 3.083 & 3.244 & & Calc. ${ }^{[15]}$ & 4.364 \\
Calc. ${ }^{[27]}$ & 3.08 & 3.27 & & Calc. ${ }^{[30]}$ & 4.363 \\
Exp. ${ }^{[28]}$ & 3.088 & 3.241 & & Exp. ${ }^{[31]}$ & 4.358 \\
Exp. ${ }^{[29]}$ & 3.10 & 3.23 & & Exp. ${ }^{[32]}$ & 4.3596 \\
\hline
\end{tabular}

\section{2 slab thickness and surface energy}


The (0001) plane of $\mathrm{TaB}_{2}$ was chosen for the fact that $\mathrm{TaB}_{2}$ is a hexagonal crystal, the (0001) plane has the highest density of atomic packing with better stability, and has been investigated extensively [27,33,34]. Considering the polar characteristics of the $\mathrm{TaB}_{2}(0001)$ plane, it well be terminated by one kind atom at one side only, thence, the $\mathrm{TaB}_{2}(0001)$ slab with the same terminal atom at two sides should be adopted to prevent the dipole effect, as presented in Fig 2 (a) and (b). Researches on SiC have shown that the (111) plane of SiC tends to be exposed to the outside ${ }^{[35,36]}$. Theoretically, $\operatorname{SiC}(111)$ slab should also consider the dipole effect caused by the difference between upper and lower surface terminal atoms. However, the study by Li et al. ${ }^{[37]}$ confirmed that the number of dangling bonds has a greater impact on the stability of $\operatorname{SiC}(111)$ surface than the dipole effect. Therefore, as presented in Fig. 2 (c) and (d), an even atomic layer SiC(111) slab with fewer dangling bonds is adopted in this work, ignoring the dipole effect.

(a)

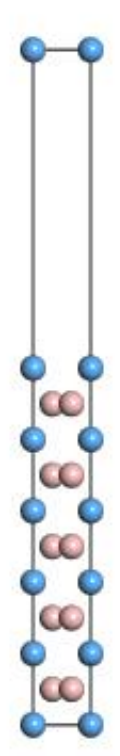

(b)

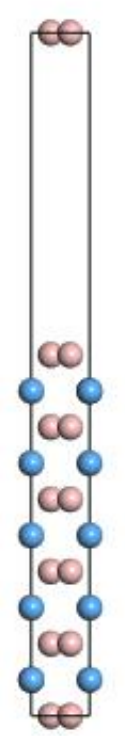

(c)

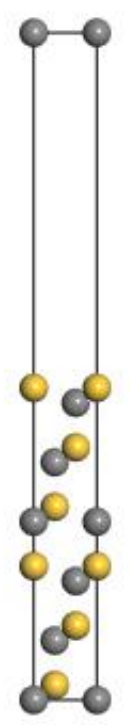

(d)

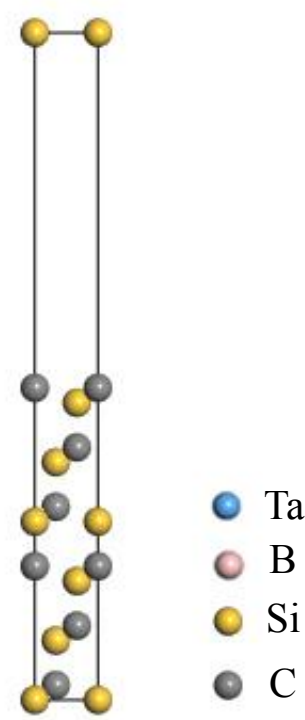

Fig. 2 Diagrammatic sketch of the $\mathrm{TaB}_{2}(0001)$ and $\mathrm{SiC}(111)$ slabs with different termination atoms, (a) and (b) are $\mathrm{TaB}_{2}(0001)$ slabs terminated by Ta and $\mathrm{B}$ atoms, respectively; (c) and (d) are $\mathrm{SiC}$ (111) slabs terminated by $\mathrm{Si}$ and $\mathrm{C}$ atoms, respectively.

The $\mathrm{TaB}_{2}(0001)$ and $\mathrm{SiC}(111)$ slabs should have sufficient thickness to achieve a bulk-like interior and ensure accurate calculation results. Undoubtedly, a slab with more atom layers could improve the accuracy of calculation results, but it will cost additional 
computational resources, so the minimum number of the atomic layers essential for the Ta or B terminated $\mathrm{TaB}_{2}(0001)$ slabs determined by observing the changing trend of the distance between adjacent atomic layers with respect to the number of atomic layers after relaxation. The alteration in atomic spacing is calculated by Eq (1):

$$
\Delta_{i j}=\frac{d_{i j}-d_{i j}^{0}}{d_{i j}^{0}} \times 100 \%
$$

Where $d_{i j}^{0}$ and $d_{i j}$ are the distances between adjacent $i$ and $j$ atomic layers before and after relaxation.

As shown in Table 2, variation of the distance between adjacent atomic layers in Taand B- terminated $\mathrm{TaB}_{2}(0001)$ surfaces are different. For the Ta terminated surface, the changes of the layer spacing mainly occur between the first and the second layer, and the variation in all slabs basically follows the contraction-expansion cycle. While for the surface ends with $\mathrm{B}$ atom, changes of the atomic layer spacing are mainly concentrated in $\triangle 2 / 3$. Overall, the variation of the interlayer distance in the interior of the Ta- and B- terminated $\mathrm{TaB}_{2}(0001)$ slabs can be ignored as the number of atomic layers greater than eleven, so the 11- layered Ta- and B- terminated $\mathrm{TaB}_{2}(0001)$ slabs are selected. For $\mathrm{SiC}(111)$ slab, numerous studies have demonstrated that $\mathrm{SiC}(111)$ slab with twelve atomic layers is sufficient to show the interior feature of the bulk $\mathrm{SiC}^{[11,13]}$.

Table 2 Variation of the spacing between adjacent atomic layers in fully relaxed Ta- and Bterminated $\mathrm{TaB}_{2}(0001)$ slabs, \pm indicates the expansion and contraction of interlayer distance,

\begin{tabular}{|c|c|c|c|c|c|c|c|}
\hline \multirow[t]{14}{*}{$\mathrm{TaB}_{2}$} & \multirow[t]{2}{*}{ Termination } & \multirow[t]{2}{*}{ Interlayer } & \multicolumn{5}{|c|}{ Number of atomic layers $(\mathrm{N})$} \\
\hline & & & 3 & 5 & 7 & 9 & 11 \\
\hline & $\mathrm{Ta}$ & $\triangle 1 / 2$ & -6.66 & -7.85 & -8.86 & -9.28 & -9.69 \\
\hline & & $\triangle 2 / 3$ & & 1.07 & 1.90 & 2.44 & 2.20 \\
\hline & & $\triangle 3 / 4$ & & 1.25 & 0.83 & -0.65 & -0.59 \\
\hline & & $\triangle 4 / 5$ & & -7.97 & 0.83 & 0.77 & 1.01 \\
\hline & & $\triangle 5 / 6$ & & & 1.84 & 0.71 & -0.12 \\
\hline & & $\triangle 6 / 7$ & & & -8.92 & -0.59 & -0.35 \\
\hline & & $\triangle 7 / 8$ & & & & 2.14 & 1.01 \\
\hline & & $\triangle 8 / 9$ & & & & -9.40 & -0.77 \\
\hline & & $\triangle 9 / 10$ & & & & & -2.08 \\
\hline & & $\triangle 10 / 11$ & & & & & -9.16 \\
\hline & $\mathrm{B}$ & $\triangle 1 / 2$ & -2.02 & 0.42 & 1.13 & 1.55 & 1.19 \\
\hline & & $\triangle 2 / 3$ & -2.32 & -2.02 & -2.44 & -3.27 & -2.86 \\
\hline
\end{tabular}




\begin{tabular}{ccccc}
\hline$\triangle 3 / 4$ & -2.14 & 0.89 & 0.65 & 0.77 \\
$\triangle 4 / 5$ & 0.59 & 0.83 & 0.53 & -0.14 \\
$\triangle 5 / 6$ & & -2.26 & 0.71 & 0.11 \\
$\triangle 6 / 7$ & & 0.95 & 0.47 & 0.12 \\
$\triangle 7 / 8$ & & & -2.91 & -0.18 \\
$\triangle 8 / 9$ & & & 1.25 & 0.77 \\
$\triangle 9 / 10$ & & & -2.50 \\
$\triangle 10 / 11$ & & & 0.89 \\
\hline
\end{tabular}

\section{2 surface energy}

The stability of a surface is closely related to the type of termination atom. Surface energy $\left(\gamma_{\text {surf }}\right)$ can be used to describe which kind of atomic-terminated surface is more stable. $\gamma_{\text {surf }}$ can be given by Eq (2):

$$
\gamma_{\text {surf }}=\frac{1}{2 \mathrm{~A}}\left(E_{\text {slab }}-\sum_{i} N_{i} \mu_{i}\right)
$$

Where $E_{\text {slab }}$ is the total energy of the slab after relaxation, $N$ and $\mu$ represent the number and chemical potential of $i$ atom, respectively, and $A$ is the surface area.

Since the atom types on the above and below surfaces of the $\mathrm{TaB}_{2}(0001)$ slab are the same, the ratio of $\mathrm{Ta}$ to $\mathrm{B}$ in $\mathrm{TaB}_{2}(0001)$ slab is not $1: 2$, which is a non-stoichiometric structure. Thus, the chemical potential of each atom must be considered. Theoretically, the fully relaxed $\mathrm{TaB}_{2}(0001)$ slab will be balanced with the bulk $\mathrm{TaB}_{2}$, then the following equations (3) and (4) will exist:

$$
\begin{gathered}
\mu_{T a B_{2}}^{\text {bulk }}=\mu_{T a}^{\text {slab }}+2 \mu_{B}^{\text {slab }} \\
\mu_{T a B_{2}}^{\text {bulk }}=\Delta H_{f}^{T a B_{2}}+\mu_{T a}^{b u l k}+2 \mu_{B}^{b u l k}
\end{gathered}
$$

Where $\mu_{T a B_{2}}^{\text {bulk }}$ is the total energy of bulk $\mathrm{TaB}_{2}, \mu_{T a}^{\text {slab }}$ and $\mu_{B}^{\text {slab }}$ are the chemical potential of Ta and $\mathrm{B}, \mu_{T a}^{\text {bulk }}$ and $\mu_{B}^{\text {bulk }}$ are the energies of a single atom in bulk Ta and B, respectively. $\Delta H_{f}^{\mathrm{TaB}_{2}}$ is the formation heat of $\mathrm{TaB}_{2}$,

According to equations (2) to (4), equation (5) will exist:

$$
\gamma_{\text {surf }}^{\text {TaB2 }}=\frac{1}{2 A}\left[E_{\text {slab }}-\frac{1}{2} N_{B} E_{T a B_{2}}^{\text {slab }}+\left(\frac{1}{2} N_{B}-N_{T a}\right)\left(\mu_{T a}^{\text {slab }}-\mu_{T a}^{b u l k}\right)+\left(\frac{1}{2} N_{B}-N_{T a}\right) \mu_{T a}^{b u l k}\right]
$$

Given that $\mathrm{TaB}_{2}$ bulk is more stable than the respective elementary substance, so the $\mu_{T a}^{\text {bulk }}$ and $\mu_{B}^{\text {bulk }}$ must be greater than $\mu_{T a}^{\text {slab }}$ and $\mu_{B}^{\text {slab }}: \mu_{B}^{\text {bulk }} \geq \mu_{B}^{\text {slab }}, \mu_{T a}^{\text {bulk }} \geq \mu_{T a}^{\text {slab }}$,

$$
\Delta \mu_{B}=\mu_{B}^{\text {slab }}-\mu_{B}^{\text {bulk }} \leq 0
$$




$$
\Delta \mu_{T a}=\mu_{T a}^{s l a b}-\mu_{T a}^{b u l k} \leq 0
$$

Consequently, the chemical potential range of the tantalum is as follows:

$$
\Delta H_{f}\left(T a B_{2}\right) \leq \mu_{T a}^{\text {slab }}-\mu_{T a}^{b u l k} \leq 0
$$

In this work, the calculated formation energy of $\Delta H_{T a B 2}=-2.067 \mathrm{eV}$, is very close to the experimental value of $2.0 \mathrm{eV}^{[38]}$ and calculation result of $-2.08 \mathrm{eV}^{[27]}$. Thus, the surface energy of the Ta and $\mathrm{B}$ terminated $\mathrm{TaB}_{2}(0001)$ surfaces can be determined, as

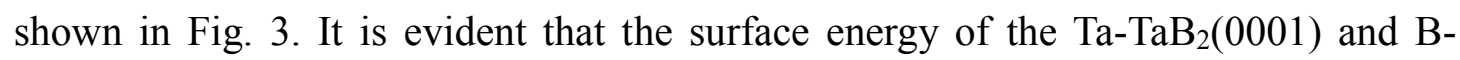
$\mathrm{TaB}_{2}(0001)$ as a function of $\Delta \mu_{T a}$. B-TaB $2(0001)$ surface has smaller surface energy than that of $\mathrm{Ta}^{-\mathrm{TaB}_{2}(0001)}$ in the Ta-poor side, and the $\gamma_{\text {surf }}$ of $\mathrm{B}-\mathrm{TaB}_{2}(0001)$ surface raised with the increase of $\Delta \mu_{\mathrm{Ta}}$, while the Ta-termination one declined, accordingly, the B-TaB $2(0001)$ surface turns into the one with larger surface energy at Ta-rich side. Smaller surface energy means a better stability, so we believe that the $\mathrm{B}^{-\mathrm{TaB}_{2}}(0001)$ surface has a better stability than $\mathrm{Ta}^{-\mathrm{TaB}_{2}}$ (0001). The higher stability of the B$\mathrm{TaB}_{2}(0001)$ surface may have a weaker interaction with other surfaces, which will be verified in the subsequent adhesion energy calculation. As presented in Fig. 2, for SiC(111), the atom types on the above and below surfaces are different, so the $\gamma_{\text {surf }}$ of the $\mathrm{SiC}(111)$ surface are calculated using the method reported in Ref. ${ }^{[30]}$. Our calculated $\gamma_{\text {surf }}$ of $4.22 \mathrm{~J} / \mathrm{m}^{2}$ is close to the other given results of $4.16 \mathrm{~J} / \mathrm{m}^{2}$ and $4.33 \mathrm{~J} / \mathrm{m}^{2}$.

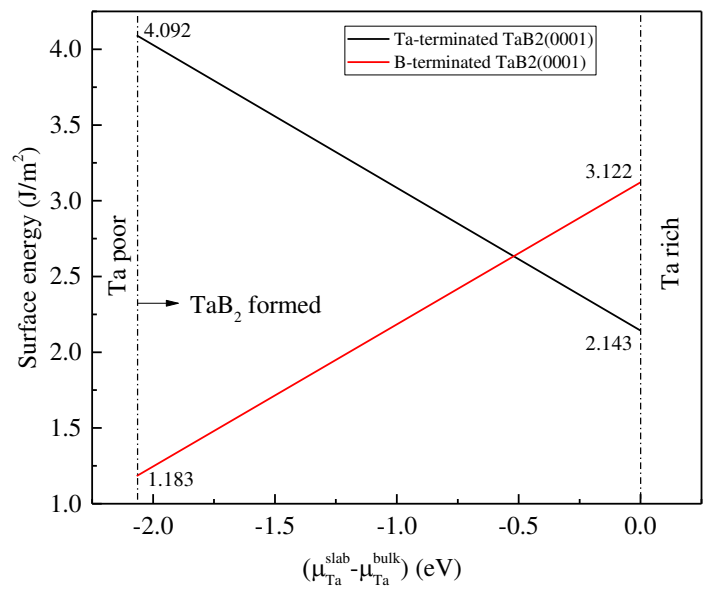

Fig. 3 Calculated surface energies of $\mathrm{TaB}_{2}(0001)$ slabs as a function of the tantalum chemical potential.

\subsection{Work of adhesion}

As presented in Fig. 4, $\mathrm{TaB}_{2}(0001) / \mathrm{SiC}(111)$ interfaces are constructed by placing 
$\mathrm{SiC}(111)$ surface on the $\mathrm{TaB}_{2}(0001)$ surface and adding a $15 \AA$ vacuum layer. Considering the difference between lattice length of the $\mathrm{TaB}_{2}(0001)\left(\mathrm{d}_{\mathrm{Ta}-\mathrm{TaB} 2(0001)}\right.$ $\left.=3.1322 \AA, \mathrm{d}_{\mathrm{B}-\mathrm{TaB} 2(0001)}=3.1334 \AA\right)$ and the $\mathrm{SiC}(111)\left(\mathrm{d}_{\mathrm{Si}-\text { or C-SiC}(111)}=3.0745 \AA\right)$, a slight tensile strain is applied to $\mathrm{SiC}(111)$ surface to match the $\mathrm{TaB}_{2}(0001)$ surface according to the method of Trivedi ${ }^{[39]}$. Then, we further assume that the interface strain can be ignored in such a coherent interface and the energetics of the interfacial bonding will not be affected.

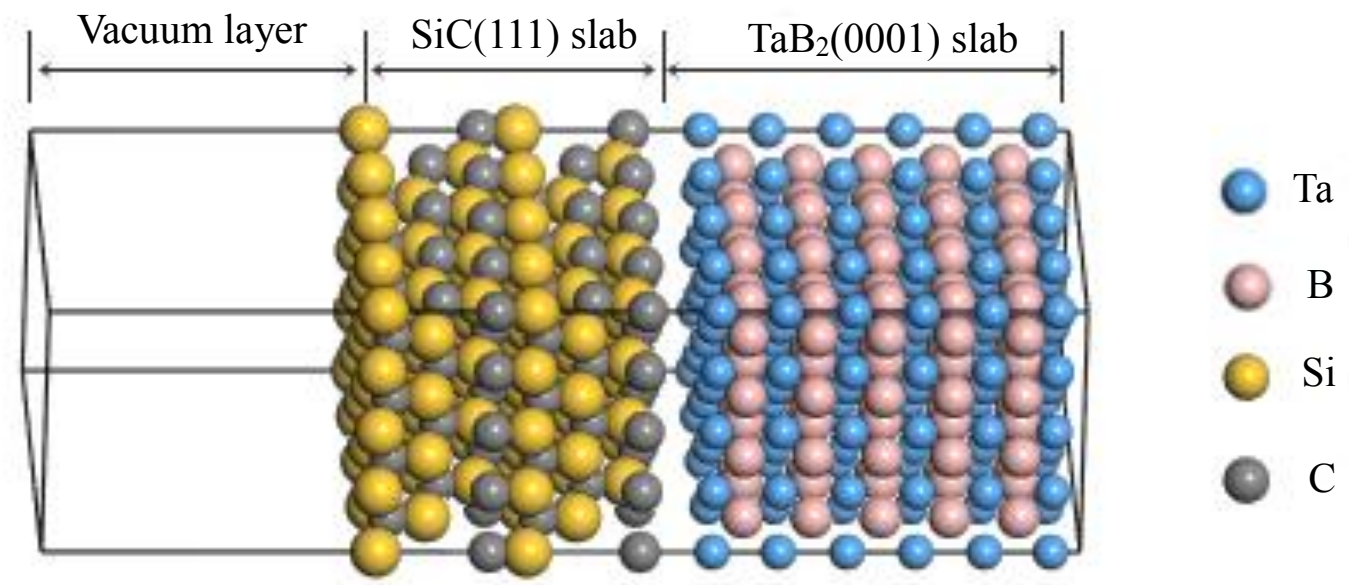

Fig. 4 Schematic diagram of $\mathrm{TaB}_{2}(0001) / \mathrm{SiC}(111)$ interface.

The interface atoms at $\mathrm{SiC}$ side have different stacking positions on $\mathrm{TaB}_{2}(0001)$ surface. As exhibited in Fig. 5, taking the interface atoms at $\mathrm{SiC}$ side being $\mathrm{C}$ atoms as an example to describe the stacking positions. Interfacial $\mathrm{C}$ atoms have three (top, center and hollow) or two (top and center) possible stacking sites on Ta-terminated and B-terminated $\mathrm{TaB}_{2}(0001)$ surface, respectively. The top site (TS) indicates that interfacial $\mathrm{C}$ atoms are situated on the top of the interfacial atoms of $\mathrm{TaB}_{2}$ side (Fig. 5 (a) and (d)); The center site (CS) indicates that interfacial C atoms are located in the center position of interfacial atoms of $\mathrm{TaB}_{2}$ side (Fig. 5 (b) and (e)); While the hollow site (HS) represents that interfacial $\mathrm{C}$ atoms are directly placed on the second layer atoms of $\mathrm{TaB}_{2}$ side, as presented in Fig. 5 (c). When the interfacial atom of SiC side is $\mathrm{Si}$, the stacking site is similar to the case of C. Thereby, totally ten interface 
configurations (Ta-(TS, CS, HS)-C, Ta-(TS, CS, HS)-Si, B-(TS, CS)-C, B-(TS, CS)-Si)

were investigated, where the first and last elements denote the terminal atom of the $\mathrm{TaB}_{2}$ and $\mathrm{SiC}$ slabs, respectively, and the middle part is the stacking position.

(a)
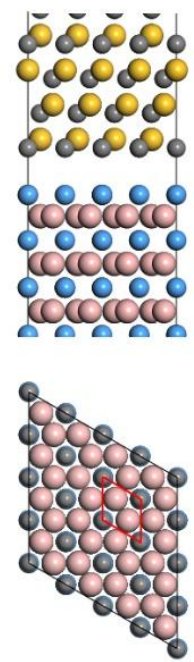

(b)
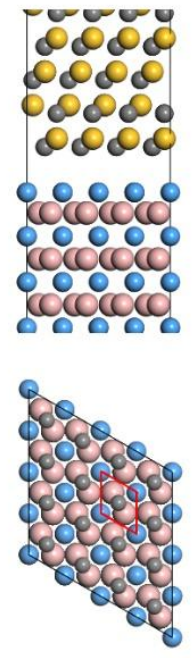

(c)
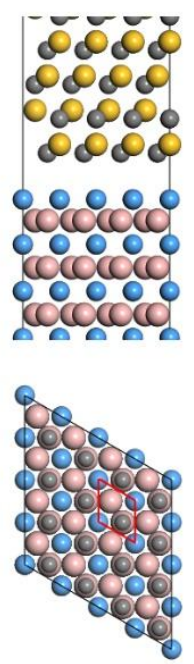

(d)
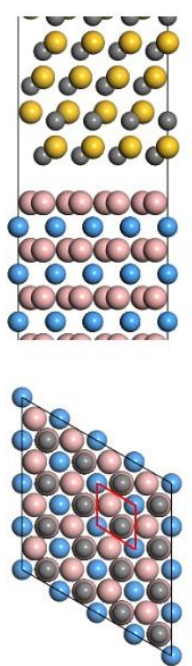

(e)

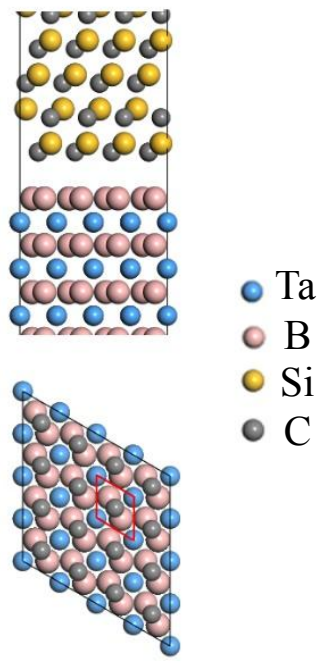

Fig. 5 Possible interfacial atomic structures of the $\mathrm{TaB}_{2}(0001) / \mathrm{SiC}(111)$ interface: (a)-(c) are the possible positions of $\mathrm{C}$ atoms on Ta-terminated $\mathrm{TaB}_{2}(0001)$ surface, (d) and (e) are the possible positions of $\mathrm{C}$ atoms on $\mathrm{B}$-terminated $\mathrm{TaB}_{2}(0001)$ surface.

For different $\mathrm{TaB}_{2} / \mathrm{SiC}$ configurations, bonding strength is the primary factor that must be considered. Bonding strength can be evaluated by the work of adhesion $\left(W_{a d}\right)$, which can be defined as the difference in energy between the system in which the surfaces are free to another in which they are in contact, forming the interface. $W_{a d}$ be determined according to Eq. (9) ${ }^{[40] \text { : }}$

$$
W_{a d}=\left(E_{\text {slab }}^{\text {SiC }}+E_{\text {slab }}^{T a B 2}-E_{\text {interface }}^{T a B 2 / S i C}\right) / A
$$

Where $E_{\text {slab }}^{S i C}$ and $E_{\text {slab }}^{T a B 2}$ denote the energies of the separated twelve-layer SiC(111) and eleven-layer $\mathrm{TaB}_{2}(0001)$ slabs with the same lattice parameter as $\mathrm{TaB}_{2} / \mathrm{SiC}$ interface, respectively. $E_{\text {interface }}^{T a B 2}$ is the energy of $\mathrm{TaB}_{2} / \mathrm{SiC}$ interface system, and $A$ refers to the interfacial area.

To determine the appropriate spacing distance $\left(d_{0}\right)$ between $\mathrm{TaB}_{2}(0001)$ and $\mathrm{SiC}(111)$ slabs, the interface distance between two slabs is manually adjusted from $0.8 \AA$ to 3.0 $\AA$, and then the relationship between separate distance $\left(d_{0}\right)$ and work of adhesion $\left(W_{a d}\right)$ 
was obtained. As it can be seen from Fig. 6, for all unrelaxed interfaces, $W_{a d}$ is a function of separation distance $\left(d_{0}\right)$ with parabolic-like. The optimal $d_{0}$ is the abscissa corresponding to the maximum value of the ordinate in each $W_{a d}$ versus $d_{0}$ profile. Generally, a larger $W_{a d}$ means higher bonding strength and better stability of the interface, so the configurations of Ta-CS-C, Ta-HS-Si, B-CS-C and B-TS-Si have better stability than other structures.
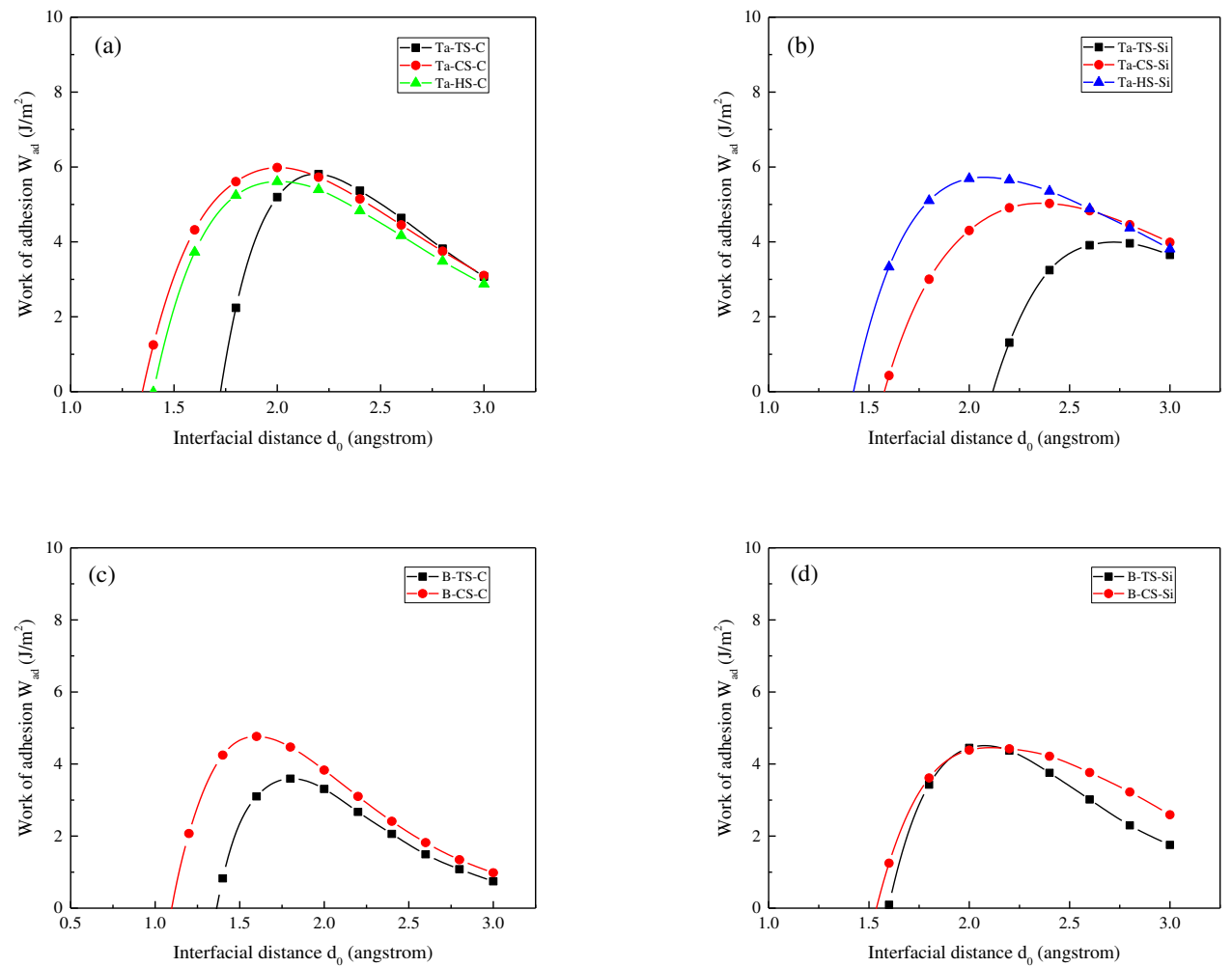

Fig. 6 Interfacial work of adhesion $\left(W_{a d}\right)$ versus spacing distance $\left(d_{0}\right)$ between $\mathrm{TaB}_{2}$ and $\mathrm{SiC}$ slabs.

The equilibrium states of the ten $\mathrm{TaB}_{2} / \mathrm{SiC}$ interfaces are also calculated by fully relaxing the interface geometries. The $W_{a d}$ and $d_{0}$ of the ten $\mathrm{TaB}_{2} / \mathrm{SiC}$ interfaces before and after relaxation are summarized in Table 3 for comparison. Difference in bonding strength of the interfaces with different stacking positions and terminal atoms can be noticed. Besides, the larger $W_{a d}$ always appears in the interface with the smaller spacing distance. All of these demonstrate that the termination atom and stacking position are the key factors affecting the bonding strength. Comparing with the unrelaxed configurations, the interface spacing becomes shorter and the bonding strength 
becomes larger after optimization, the reduced distance between $\mathrm{TaB}_{2}$ and $\mathrm{SiC}$ slabs thereby enhancing their interaction. This is the reason why the $W_{a d}$ of all interfaces becomes larger after relaxation. It is worth noting that the $W_{a d}$ of the interfaces with B termination at $\mathrm{TaB}_{2}$ side is smaller than the Ta-terminated ones, which is consistent with the $\mathrm{B}-\mathrm{TaB}_{2}(0001)$ surface energy analysis result. Among them, the relaxed Ta-CS-C, Ta-HS-Si, B-CS-C and B-TS-Si configurations exhibit the largest $W_{a d}$ of $6.04 \mathrm{~J} / \mathrm{m}^{2}, 5.84$ $\mathrm{J} / \mathrm{m}^{2}, 4.73 \mathrm{~J} / \mathrm{m}^{2}$ and $4.47 \mathrm{~J} / \mathrm{m}^{2}$, respectively.

Table 3 Interfacial spacing distance $d_{0}$ and bonding strength between $\mathrm{TaB}_{2}$ and $\mathrm{SiC}$ slabs in all configurations before and after relaxation.

\begin{tabular}{cccccccc}
\hline \multirow{2}{*}{$\mathrm{TaB}_{2}(0001)$} & \multirow{2}{*}{$\mathrm{SiC}(111)$} & Stacking & Interface & \multicolumn{2}{c}{ Unrelaxed } & \multicolumn{2}{c}{ Relaxed } \\
\cline { 5 - 8 } & & sites & model & $d_{0}$ & $W_{a d}$ & $d_{0}$ & $W_{a d}$ \\
\hline \multirow{2}{*}{ Ta-termination } & C-termination & Top & Ta-TS-C & 2.20 & 5.24 & 2.190 & 5.98 \\
& & Center & Ta-CS-C & 2.00 & 5.41 & 1.977 & 6.04 \\
& & Hollow & Ta-HS-C & 2.00 & 5.04 & 1.857 & 5.83 \\
& \multirow{4}{*}{ Si-termination } & Top & Ta-TS-Si & 2.80 & 3.39 & 2.697 & 4.16 \\
& & Center & Ta-CS-Si & 2.40 & 4.45 & 2.382 & 5.07 \\
& & Hollow & Ta-HS-Si & 2.00 & 5.12 & 1.863 & 5.84 \\
B-termination & C-termination & Top & B-TS-C & 1.80 & 3.53 & 1.656 & 3.61 \\
& & Center & B-CS-C & 1.60 & 4.70 & 1.595 & 4.73 \\
& \multirow{2}{*}{ Si-termination } & Top & B-TS-Si & 2.10 & 4.45 & 2.059 & 4.47 \\
& & Center & B-CS-Si & 2.10 & 4.36 & 2.054 & 4.43 \\
\hline
\end{tabular}

\subsection{Interfacial energy}

Interfacial energy $\left(\gamma_{\text {int }}\right)$ is always employed to describe the thermodynamic stability of an interface, and can be regarded as the difference between surface energies of two slabs, and the work of adhesion. The lower interfacial energy implies the interface is energetically more favorable. $\gamma_{i n t}$ can be computed by ${ }^{[41]}$ :

$$
\gamma_{\text {int }}=\gamma_{T a B 2}^{\text {surf }}+\gamma_{\text {Sic }}^{\text {surf }}-W_{a d}
$$

Where $\gamma_{\text {TaB2 }}^{\text {surf }}$ and $\gamma_{\text {SiC }}^{\text {surf }}$ denote the $\mathrm{TaB}_{2}(0001)$ and $\mathrm{SiC}(111)$ slab surface energy, respectively, and $W_{a d}$ is the work of adhesion.

The relationship between interfacial energy and $\mu_{T a}^{\text {slab }}-\mu_{T a}^{b u l k}$ of the ten $\mathrm{TaB}_{2} / \mathrm{SiC}$ interfaces were shown in Fig. 7, and the $\gamma_{\text {int }}$ values of each configuration were summarized in Table 4. Clearly, the interfacial energies as a function of $\mu_{T a}^{\text {slab }}-\mu_{T a}^{b u l k}$ : within the range of $\Delta \mu_{\mathrm{Ta}}$, the interfacial energies of the interfaces with Ta termination 
at $\mathrm{TaB}_{2}$ side decrease monotonously, which is opposite to that of $\mathrm{B}$ terminated interfaces. The Ta-CS-C and B-CS-C interfaces have the lowest interfacial energies of $0.323 \sim 2.272 \mathrm{~J} / \mathrm{m}^{2}$ and $0.313 \sim 2.612 \mathrm{~J} / \mathrm{m}^{2}$, respectively. Combined with the calculation results of the $W_{a d}$, it can be determined that the Ta-CS-C and B-CS-C are the most preferred and stable configurations due to their smallest interfacial energy and largest adhesion energy.

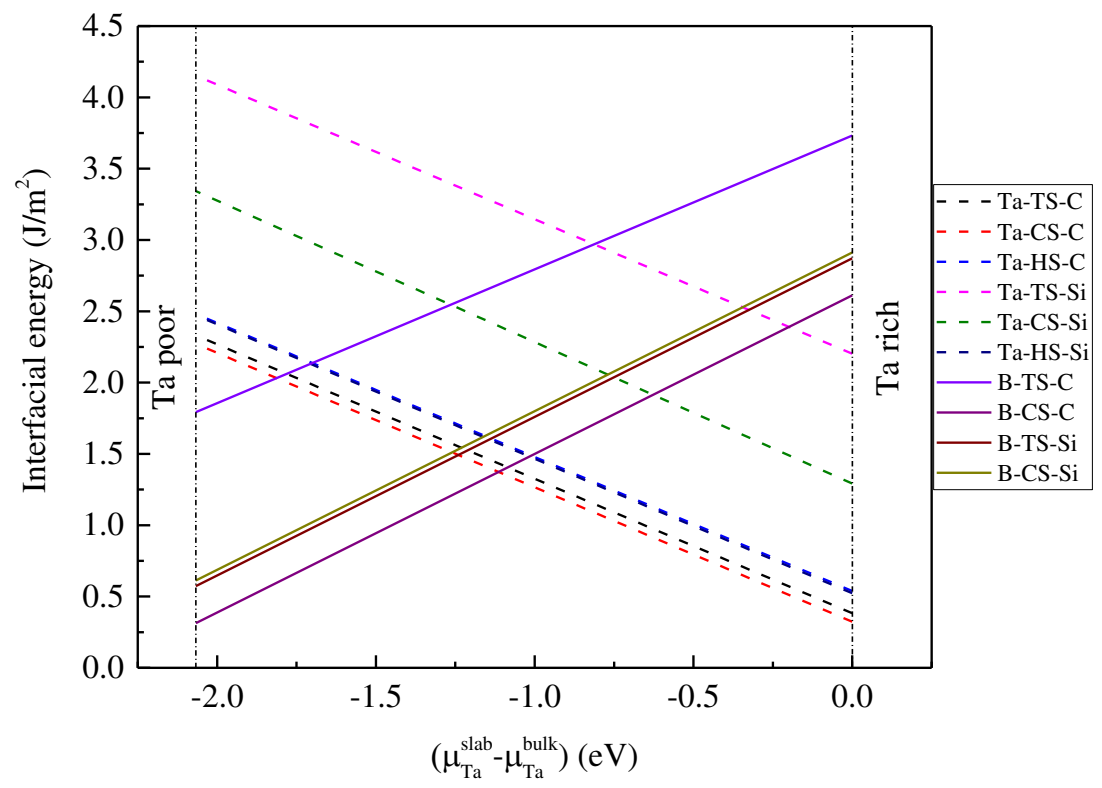

Fig. 7 Interfacial energies of the ten $\mathrm{TaB}_{2} / \mathrm{SiC}$ interfaces versus chemical potential of tantalum.

\begin{tabular}{cc} 
Table 4 Interfacial energy values of each interface configura \\
\hline Configuration & Interfacial energy $\left(\mathrm{J} / \mathrm{m}^{2}\right)$ \\
\hline Ta-TS-C & $0.383 \sim 2.332$ \\
Ta-CS-C & $0.323 \sim 2.272$ \\
Ta-HS-C & $0.538 \sim 2.482$ \\
Ta-TS-Si & $2.203-4.152$ \\
Ta-CS-Si & $1.293 \sim 3.242$ \\
Ta-HS-Si & $0.523 \sim 2.472$ \\
B-TS-C & $1.793 \sim 3.372$ \\
B-CS-C & $0.313 \sim 2.612$ \\
B-TS-Si & $0.573 \sim 2.872$ \\
B-CS-Si & $0.613 \sim 2.912$ \\
\hline
\end{tabular}

\subsection{Electronic structure}

The stability and mechanical properties of the Ta-CS-C and B-CS-Cstructures are 
closely related to the interfacial electronic interaction. Therefore, calculation of the charge density distribution, charge density difference and partial density of states (PDOS) were performed. Fig. 8 shows the charge density distribution ((a) and (b)) and charge density differences ((c) and (d)) of the Ta-CS-C and B-CS-C interfaces, respectively. It can be seen from Fig. 8 (a) and (b) that the Ta-C chemical bonds formed between interfacial $\mathrm{C}$ and Ta atoms in Ta-CS-C, while the C-B chemical bonds formed between interfacial $\mathrm{C}$ and $\mathrm{B}$ atoms in B-CS-C. Inspecting Fig. 8 (c) that the electron density between $\mathrm{C}$ and $\mathrm{Ta}$ is small, and the partial valence electron of the interfacial Ta in the direction $\mathrm{C}$ and $\mathrm{Ta}$ are lost, which is the characteristic of ionic bonds. For the BCS-C interface (Fig. 8 (d)), the charge transfer characteristics is completely different from the Ta-CS-C, a significant charge accumulated at the interstitial region between interfacial $\mathrm{C}$ and $\mathrm{B}$ atoms, indicating that typical covalent bonds formed between $\mathrm{B}$ and $\mathrm{C}$ in B-CS-C interface.

(a)

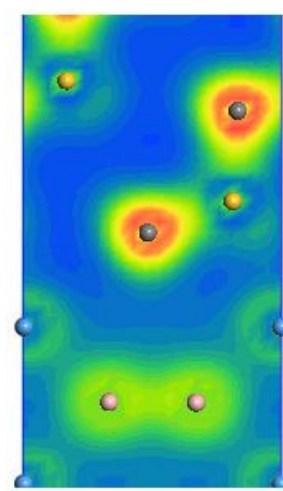

(c)

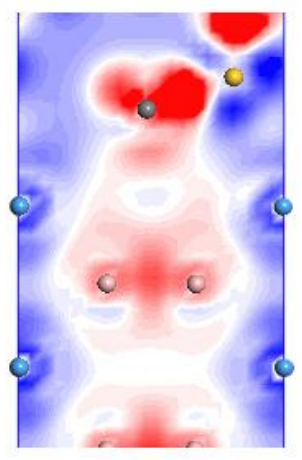

(b)

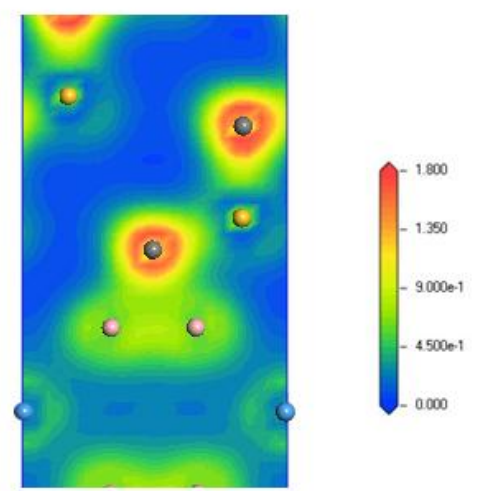

(d)

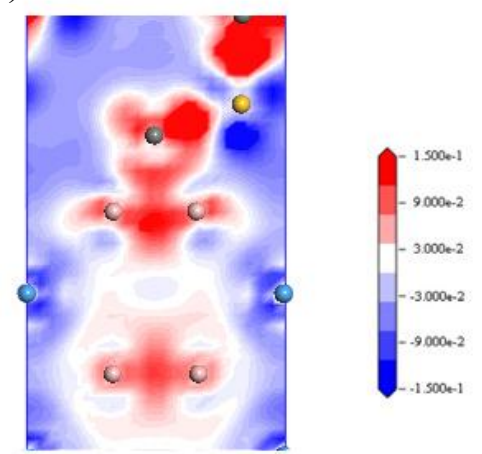

Fig. 8 Charge density distribution ((a) and (b)) and charge density difference((c) and (d)) through (110) for Ta-CS-C and B-CS-C interfaces. 
For the purpose of further illustrating the electronic interaction and bonding feature of the Ta-CS-C and B-CS-C interfaces, the layer-projected partial density of states (LPDOS) is performed, as shown in Fig. 9. A grid meshing of $21 \times 21 \times 1$ was applied to improve the accuracy. Noting that the total density of states (TDOS) curves of the TaCS-C and B-CS-C interfaces are very similar, which means that they have similar electronic structures. Besides, both Ta-CS-C and B-CS-C interfaces have metallic properties, this can be proved by the peak of TDOS curve at Fermi level.

For the Ta-CS-C interface (Fig. 9 (a)), the orbital hybridization of the interfacial C-p and Ta- $d$ orbits was observed in the range of $-10 \mathrm{eV} \sim-0 \mathrm{eV}$, the density of states peaks of interfacial C- $p$ in this range are sharper than that of inner atoms, while Ta- $d$ are flatter, which means that the ionic bonds exist between interfacial $\mathrm{Ta}$ and $\mathrm{C}$ atoms. Compared with the $3^{\text {rd }} \mathrm{C}$ and Ta atoms, the interfacial C-s have a higher state at $-12 \mathrm{eV}$, while the interfacial Ta- $d$ have a lower state at here, which also confirms the existence of ionic bond. In addition, the interfacial C- $p$ and Ta- $d$ have the larger DOS values than those of inner atoms in the range from $-1.5 \mathrm{eV}$ to Fermi level, which indicates that they also have certain metallic bond component. Therefore, the ionic and metallic bonds coexist in Ta-CS-C interface. As for the B-CS-C interface (Fig. 9 (b)), due to the interaction of interfacial atoms, the partial density state curve of the atoms near the interface is different from that of internal atoms, especially the interfacial $\mathrm{C}$ and $\mathrm{B}$ atoms. The obvious overlapping state of interfacial $\mathrm{C}$ and $\mathrm{B}$ atoms can be found in the range of -12 $\mathrm{eV}$ to $0 \mathrm{eV}$, and the identical peaks appear at $-10.5 \mathrm{eV},-6.7 \mathrm{eV},-4 \mathrm{eV}$ and $-1 \mathrm{eV}$, indicating that covalent bonds formed between interfacial C and B atoms. Meanwhile, it is also noticed that the $d$ orbital of the $2^{\text {nd }}$ layer Ta atoms of $\mathrm{TaB}_{2}$ side is significantly different from the $4^{\text {th }}$ layer Ta atoms in the vicinity of the Fermi level, and a peak corresponding to the interfacial $\mathrm{C}$ and $\mathrm{B}$ atoms appear at $-1 \mathrm{eV}$, which proves that covalent bond also formed between interfacial $\mathrm{C}$ atoms of $\mathrm{SiC}$ side between $2^{\text {nd }}$ layer Ta atoms. In all, covalent bond played a dominant role in B-CS-C interface. 
(a)

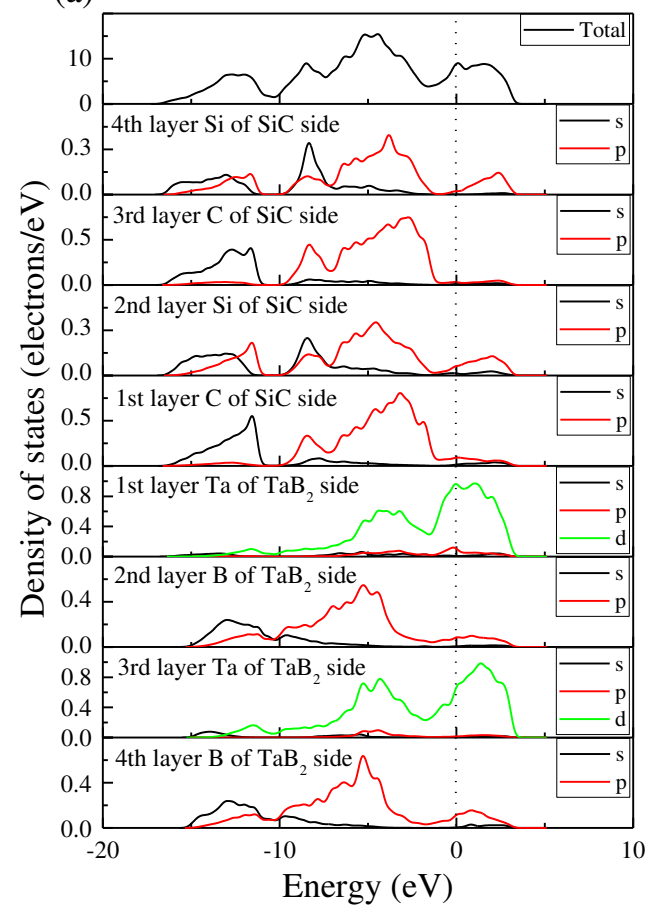

(b)

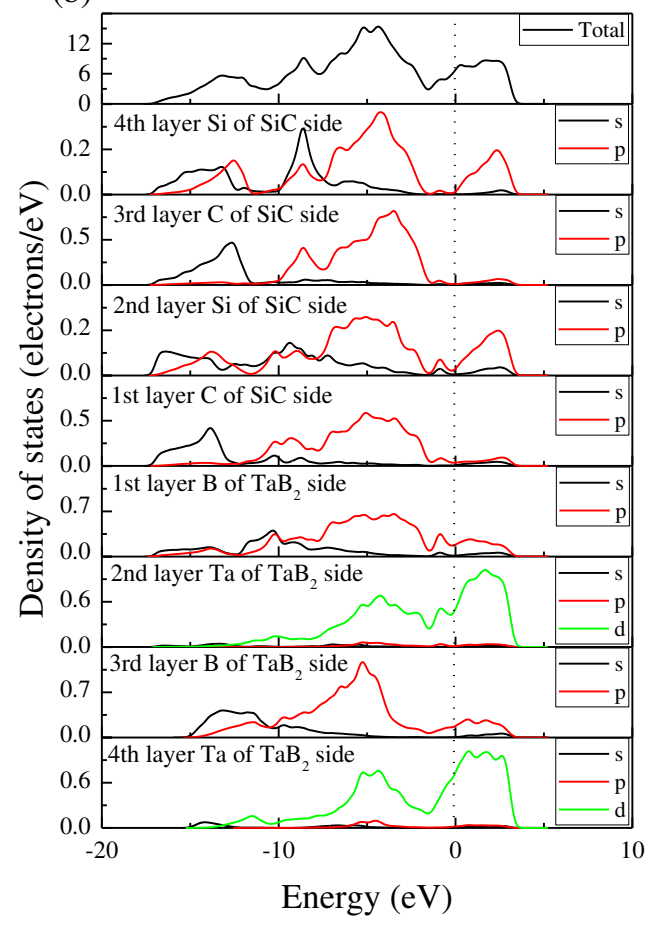

Fig. 9 Layer-projected partial density of states (LPDOS) for relaxed $\mathrm{TaB}_{2}(0001) / \mathrm{SiC}(111)$ interfaces. (a) Ta-CS-C; (b) B-CS-C. The dotted line indicates the location of the Fermi level.

\section{Conclusions}

In this present work, ten $\mathrm{TaB}_{2} / \mathrm{SiC}$ interfaces were constructed in the consideration of different terminations and stacking sites. The atomic structure, bonding strength, stability and electronic feature of the $\mathrm{TaB}_{2}(0001) / \mathrm{SiC}(111)$ interfaces were analyzed by examining the adhesion energy, interfacial energy, and bonding nature. With the help of first-principles study, it was found that termination atom and stacking position are the key factors affecting the bonding strength and stability of an interface, the Ta$\mathrm{TaB}_{2} / \mathrm{C}$-SiC centre-site stacked (Ta CS-C) and B- $\mathrm{TaB}_{2} / \mathrm{C}-\mathrm{SiC}$ center-site stacked (BCS-C) configurations are significantly more stable than any others, which are the most preferred interface structures. By performing the charge density difference and partial density of states (PDOS) analysis, electronic interactions in Ta-CS-C and B-CS-C interfaces were revealed, and confirming that ionic and metallic bonds coexisted in TaCS-C interface, while covalent bond played a dominant role in B-CS-C interface. In all, comparing the bonding strength and stability of each interface, we believe that Ta-CS$\mathrm{C}$ and $\mathrm{B}-\mathrm{CS}-\mathrm{C}$ interfaces are more likely to appear in the $\mathrm{TaB}_{2}-\mathrm{SiC}$ coating. 


\section{Acknowledgments}

This work was sponsored by the Foundation Strengthening Program of China (No. 173205), by Basic Research on Protective Coating Technology of Hot-end Components (No. 2017-JCJQ-ZD-031).

\section{References}

[1] Yingjie Xu, Pan Zhang, Huan Lu, Weihong Zhang, Hierarchically modeling the elastic properties of 2D needled carbon/carbon composites, Compos. Struct., 133 (2015) 148-156.

[2] Nathan S. Jacobson, Donald M. Curry, Oxidation microstructure studies of reinforced carbon/carbon, Carbon 44 (2006) 1142-1150.

[3] Ahmed Sameer, Khan Mohammed, Huseyin Sehitoglu, Richard Rateick, Interface graphitization of carbon-carbon composites by nanoindentation, Carbon 150 (2019) 425-435.

[4] Tao Li, Yulei Zhang, Jia Sun, Jinrui Ren, et al. In situ synthesis of SiC nanowire porous layer on carbon/carbon composites, J. Am. Ceram. Soc., 101(2018):1371-1380. [5] Ya-lei Wang, Xiang Xiong, Guo-dong Li, Huai-fei Liu, et al. Preparation and ablation properties of $\mathrm{Hf}(\mathrm{Ta}) \mathrm{C}$ co-deposition coating for carbon/carbon composites, Corros. Sci., 66 (2013) 177-182.

[6] Guo-Bin Zheng, Hideaki Sano, Yasuo Uchiyama, A carbon nanotube-enhanced SiC coating for the oxidation protection of $\mathrm{C} / \mathrm{C}$ composite materials, Compos. Part B-Eng., 42 (2011) 2158-2162.

[7] Xiaohong Shi, Xiuxiu Jin, Hongjiao Lin, Junyi Jing, et al. Joining of SiC nanowirestoughened $\mathrm{SiC}$ coated $\mathrm{C} / \mathrm{C}$ composites and nickel-based superalloy (GH3044) using Ni71CrSi interlayer, J. Alloy Comp., 693 (2017) 837-42.

[8] Xinfa Qiang, Hejun Li, Yulei Zhang, Zhangzhong Wang, et al. Mechanical and oxidation protective properties of $\mathrm{SiC}$ nanowires-toughened $\mathrm{SiC}$ coating prepared insitu by a CVD process on C/C composites, Surf. Coat. Technol., 307 (2016) 91-98.

[9] Chu Yanhui, Li Hejun, Fu Qiangang, Wang Haipeng, et al. Oxidation protection of $\mathrm{C} / \mathrm{C}$ composites with a multilayer coating of $\mathrm{SiC}$ and $\mathrm{Si}+\mathrm{SiC}+\mathrm{SiC}$ nanowires, Carbon 50 (2012) 1280-1288.

[10] Jixiang Dai, Jianjun Sha, Junqi Shao, Yufei Zu, et al. In-situ growth of SiC nanostructures and their influence on anti-oxidation capability of $\mathrm{C} / \mathrm{SiC}$ composites, Corros. Sci. 124 (2017) 71-79.

[11] Peipei Wang, Mingde Tong, Hanhui Wang, Hejun Li, et al. Gradient $\mathrm{HfB}_{2}-\mathrm{SiC}$ multilayer oxidation resistant coating for $\mathrm{C} / \mathrm{C}$ composites, Ceram. Int., 44 (2018) 20968-20973.

[12] Xuanru Ren, Hejun Li, Yanhui Chu, Kezhi Li, et al., $\mathrm{ZrB}_{2}-\mathrm{SiC}$ gradient oxidation protective coating for carbon/carbon composites, Ceram. Int., 40 (2014) 7171-7176. 
[13] Zhaoqian Li, Hejun Li, Wei Li, Jie Wang, et al., Preparation and ablation properties of $\mathrm{ZrC}-\mathrm{SiC}$ coating for carbon/carbon composites by solid phase infiltration, Appl. Surf. Sci., 258 (2011) 565-571.

[14] Yan Jiang, Tianyu Liu, Hongqiang Ru, Wei Wang, et al., Ultra-high-temperature ceramic $\mathrm{TaB}_{2}-\mathrm{SiC}$-Si coating by impregnation and in-situ reaction method to prevent graphite materials from oxidation and ablation, Ceram. Int., 45 (2019) 6541-6551.

[15] Huihui Xiong, Zhao Liu, Henghua Zhang, Zheng Du, et al., First principles calculation of interfacial stability, energy and electronic properties of $\mathrm{SiC} / \mathrm{ZrB}_{2}$ interface, J. Phys. Chem. Solids, 107 (2017) 162-169

[16] J. Tolle, J. Kouvetakis, D.-W. Kim, S. Mahajan, et al., Epitaxial growth of $\mathrm{ZrB}_{2}(0001)$ on $\mathrm{Si}(111)$ for III-nitride applications: A review, Chinese J. Phys., 43 (2005) 233-248.

[17] Po-Liang Liu, A. V. G. Chizmeshya, John Kouvetakis, Structural, electronic, and energetic properties of $\mathrm{SiC}[111] / \mathrm{ZrB}_{2}[0001]$ heterojunctions: A first-principles density functional theory study, Phys. Rev B, 77 (2008) 035326-035335.

[18] Gong Cheng, Yuqing Xiong, Hui Zhou, Kaifeng Zhang, et al., Insight into the structure stability and bonding nature of $\mathrm{HfB}_{2}(0001) / \mathrm{SiC}(111)$ interface: A firstprinciples study, Ceram. Int., https://doi.org/10.1016/j.ceramint.2020.10.010

[19] Gong Cheng, Yuqing Xiong, Hui Zhou, Kaifeng Zhang, et al., Revealing the adhesion, stability and electronic structure of $\mathrm{ZrC}(111) / \mathrm{SiC}(111)$ interface: A firstprinciples study, Vacuum, 180 (2020) 109591-109601.

[20] M D Segall, Philip J D Lindan, M J Probert, C J Pickard, et al., First-principles simulation: ideas, illustrations and the CASTEP code, J. Phys-Condens Mat., 14 (2002) 2717-2744.

[21] John P. Perdew, Kieron Burke, Matthias Ernzerhof, Generalized Gradient Approximation Made Simple, Phys. Rev. Lett., 77 (1996) 3865-3868.

[22] John P. Perdew, Kieron Burke, Yue Wang, Generalized gradient approximation for the exchange-correlation hole of a many-electron system, Phys. Rev. B, 54 (1996) 16533-16539.

[23] David Vanderbilt, Soft self-consistent pseudopotentials in a generalized eigenvalue formalism, Phys. Rev. B, 41 (1990) 7892-7895.

[24] Thomas H. Fischer and Jan Almlof, General methods for geometry and wave function optimization, J. Phys. Chem., 96 (1992) 9768-9774.

[25] Bernd G. Pfrommer, Michel Côté, Steven G. Louie, Marvin L. Cohen, Relaxation of crystals with the Quasi-Newton method, J. Comput. Phys., 131 (1997) 233-0240.

[26] S. Okada, K. Kudo, I. Higashi, T. Lundström, Single crystals of TaB, Tas $\mathrm{B}_{6}, \mathrm{Ta}_{3} \mathrm{~B}_{4}$ and $\mathrm{TaB}_{2}$, as obtained from high-temperature metal solutions, and their properties, $\mathrm{J}$. Cryst. Growth, 128 (1993) 1120-1124.

[27] Kazuo Yamamoto, Difference in the outermost layer between $\mathrm{TaB}_{2}(0001)$ and $\mathrm{HfB}_{2}(0001)$, Phys. Rev. B, 60 (1999) 15617-15620.

[28] P. Villars, Pearson's Handbook: Crystallographic Data for Intermetallic Phases, 
ASM International, Materials Park, OH, 1997.

[29] R. W. G. Wyckoff, Crystal Structures (Interscience, New York, 1965), Vol. 1.

[30] N. Jin, Y. Yang, J. Li, X. Luo, et al., First-principles calculation on $\beta-\mathrm{SiC}(111) / \alpha-$ WC(0001) interface, J. Appl. Phys., 115 (2014) 223714-223725.

[31] Z. Li, R.C. Bradt, Thermal expansion of the cubic (3c) polytype of SiC, J. Mater. Sci. 21 (1986) 4366-4368.

[32] Landolt-Börnstein New Series, in: H. Ullmeier (Eds) Numerical Data and Functional Relation-ships in Science and Technology, Springer, Hei-delberg, 2001

[33] Alexandra Evstigneeva, Rasdip Singh, Michael Trenary, Shigeki Otani, Reaction of $\mathrm{O}_{2}$ with the boron-terminated $\mathrm{TaB}_{2}$ (0001) surface, Surf. Sci., 542 (2003) 221-229.

[34] Dongliang Liu, Jianguo Deng, Yongzhong Jin, Interactions of water molecule with $\mathrm{HfB}_{2}$ and $\mathrm{TaB}_{2}(0001)$ surfaces: A first-principles investigation, Comp. Mater. Sci. 97 (2015) 116-120.

[35] Dong-Joo Kim, Doo-Jin Choi, Young-Wook Kim, Effect of reactant depletion on the microstructure and preferred orientation of polycrystalline $\mathrm{SiC}$ films by chemical vapor deposition, Thin Solid Films, 266 (1995) 192-197.

[36] Boris Reznik, Dagmar Gerthsen, Weigang Zhang, et al., Microstructure of SiC deposited from methyltrichlorosilane, J. Eur. Ceram. Soc., 23 (2003) 1499-1508.

[37] Jian Li, Yanqing Yang, Guanghai Feng, Xian Luo, et al., First-principles study of stability and properties on $\beta$-SiC/TiC(111) interface, J. Appl. Phys., 114 (2013) 163522163533

[38] N. N. Greenwood, in Comprehensive Inorganic Chemistry, edited by J. C. Bailar, H. J. Emeleus, Sir R. Nyholm, and A. F. Trotman-Dickenson (Pergamon, Oxford, 1973), Vol. 1, p. 697.

[39] Rahul Trivedi, Po-Liang Liu, Radek Roucka, et al., Mismatched heteroepitaxy of tetrahedral semiconductors with $\mathrm{Si}$ via $\mathrm{ZrB}_{2}$ templates. Chem. Mater., 17 (2005) 4647 4652.

[40] Donald J Siegel, Louis G Hector Jr., James B Adams, Adhesion, stability, and bonding at metal/metal-carbide interfaces: Al/WC, Surf. Sci., 498 (2002) 321-336.

[41] Mikael Christensen, Sergey Dudiy, and Göran Wahnström, First-principles simulations of metal-ceramic interface adhesion: $\mathrm{Co} / \mathrm{WC}$ versus $\mathrm{Co} / \mathrm{TiC}$, Phys. Rev. B, 65 (2002) 045408-045417. 
Figures

(a)

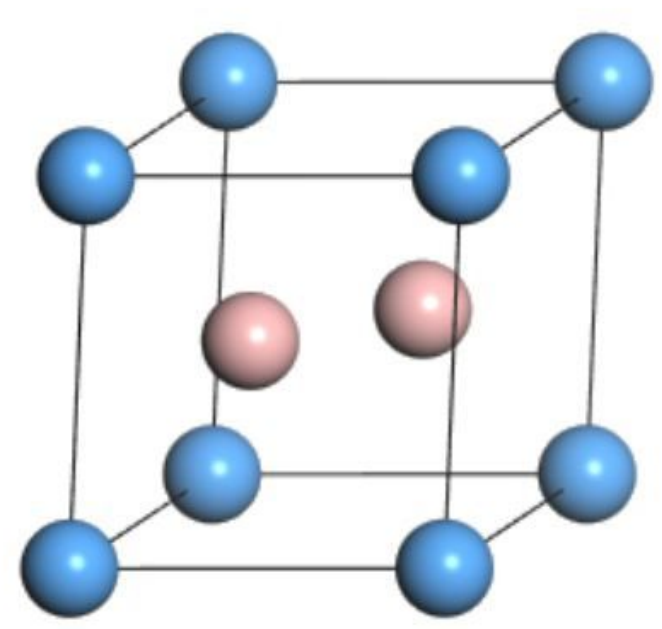

(b)

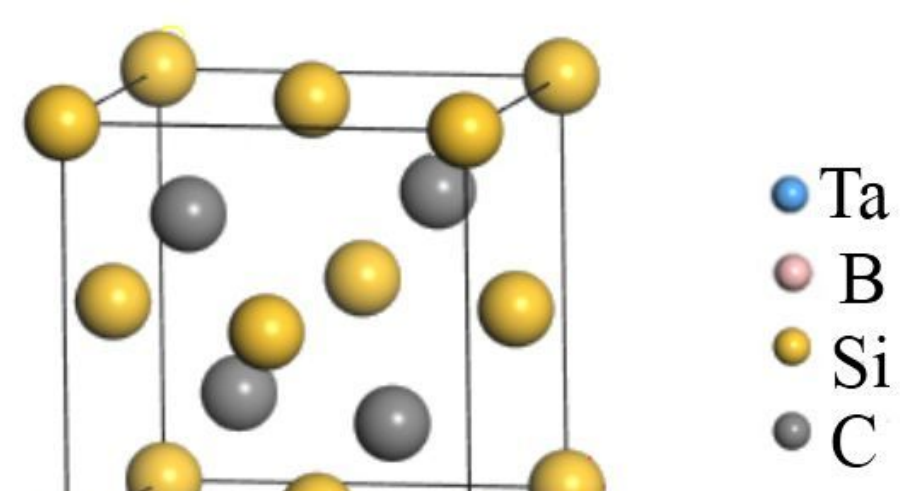

Figure 1

The crystal structures of TaB2 (a) and SiC (b).

(a)

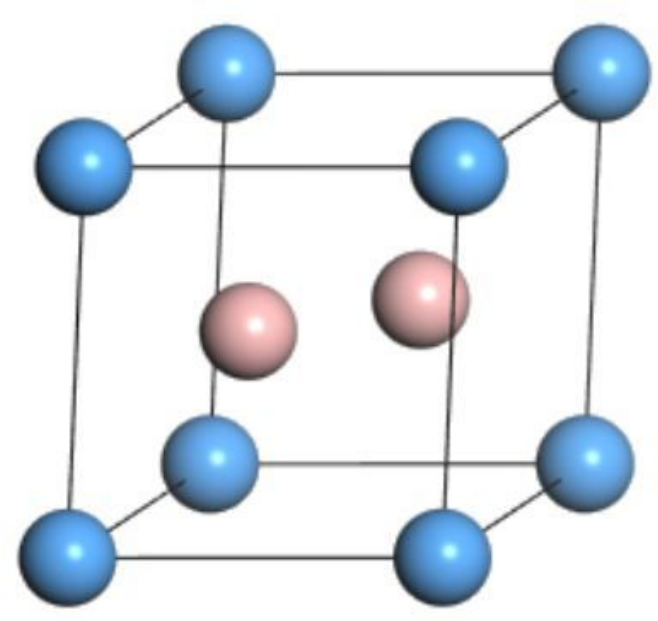

(b)

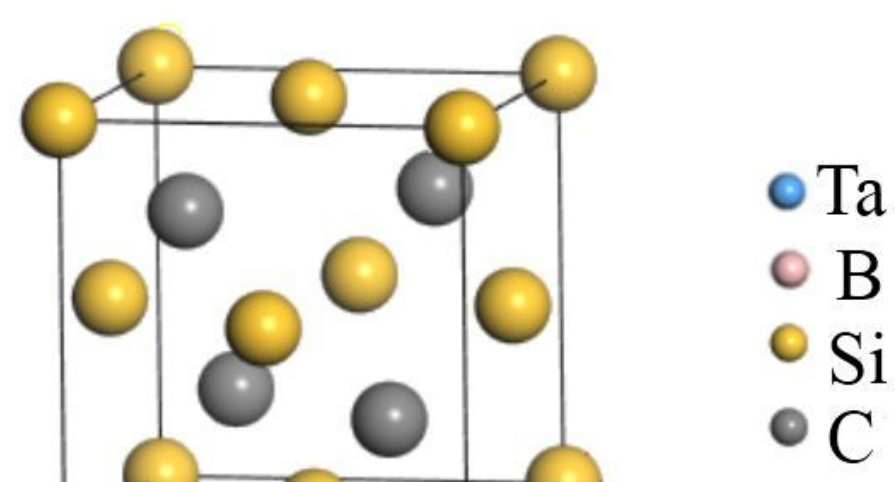

Figure 1

The crystal structures of TaB2 (a) and SiC (b). 
(a)

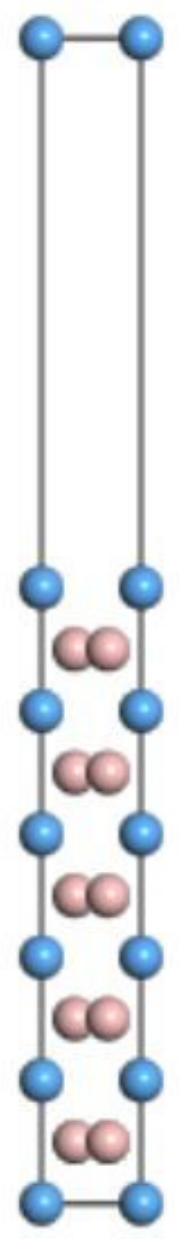

(b)

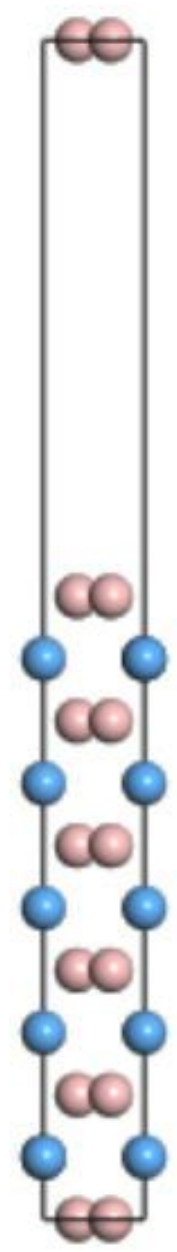

(c)

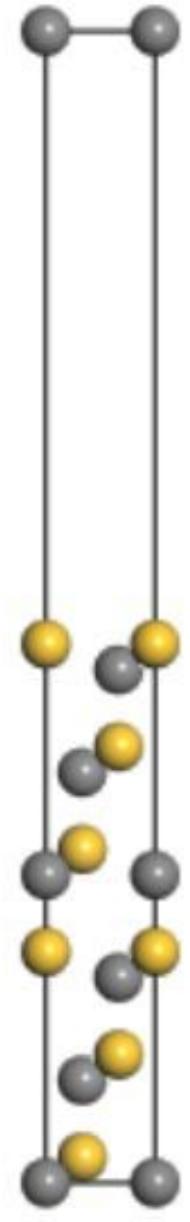

(d)

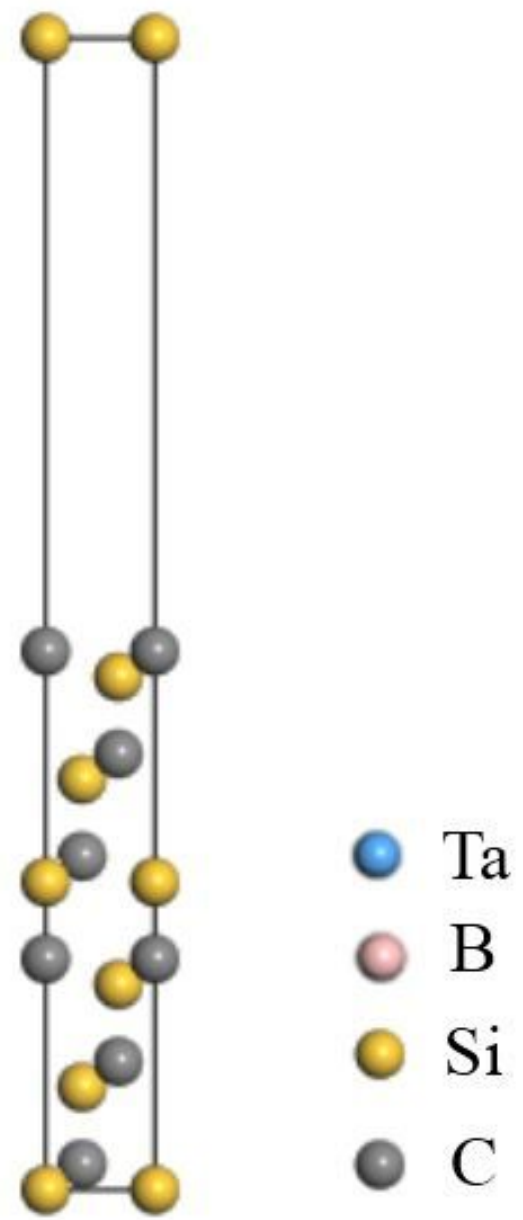

Figure 2

Diagrammatic sketch of the TaB2(0001) and $\mathrm{SiC}(111)$ slabs with different termination atoms, (a) and (b) are TaB2(0001) slabs terminated by Ta and B atoms, respectively; (c) and (d) are SiC (111) slabs terminated by $\mathrm{Si}$ and $\mathrm{C}$ atoms, respectively. 
(a)

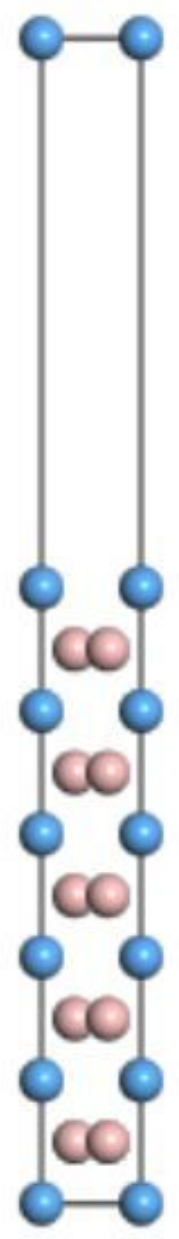

(b)

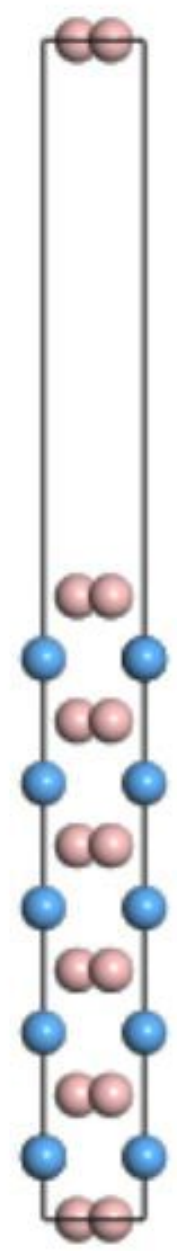

(c)

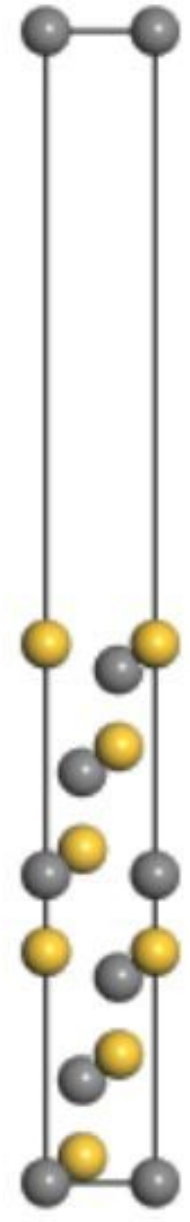

(d)

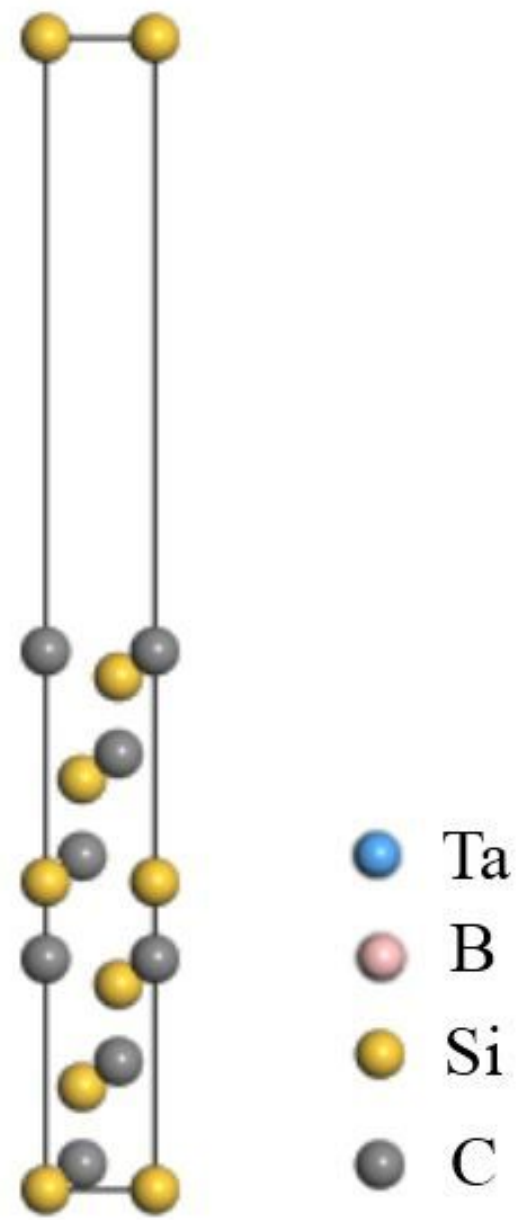

Figure 2

Diagrammatic sketch of the TaB2(0001) and $\mathrm{SiC}(111)$ slabs with different termination atoms, (a) and (b) are TaB2(0001) slabs terminated by Ta and B atoms, respectively; (c) and (d) are SiC (111) slabs terminated by $\mathrm{Si}$ and $\mathrm{C}$ atoms, respectively. 


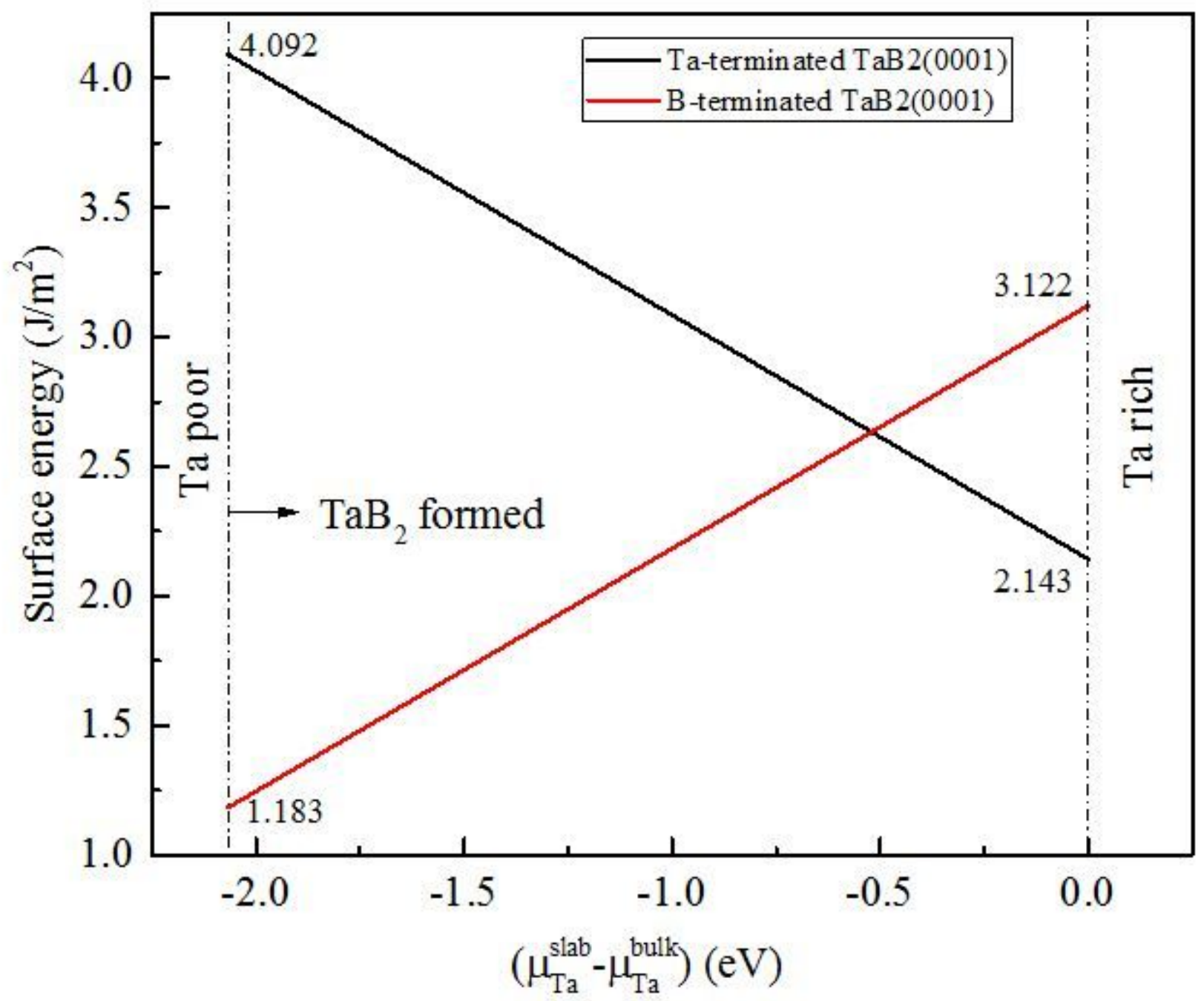

Figure 3

Calculated surface energies of TaB2(0001) slabs as a function of the tantalum chemical potential. 


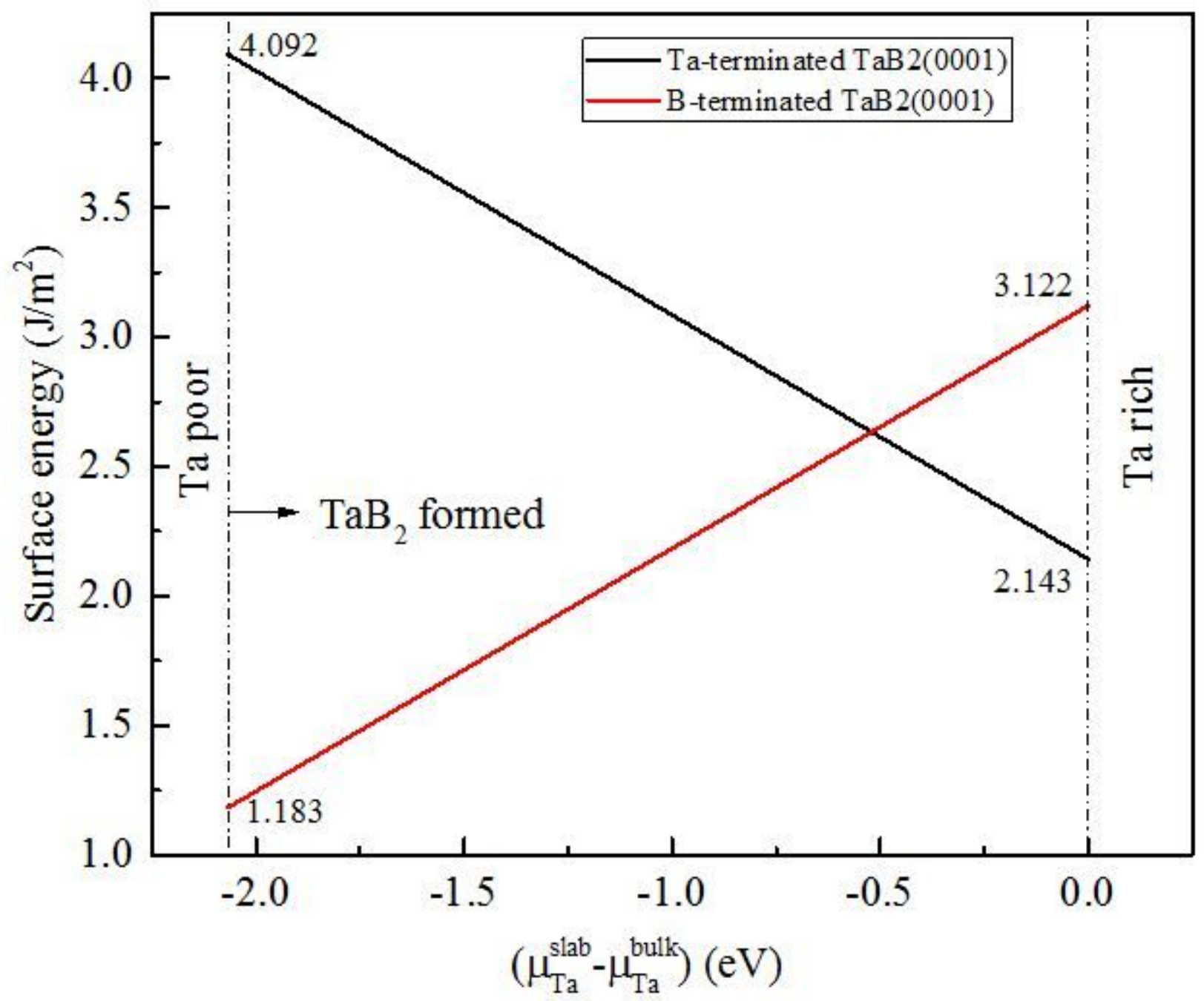

Figure 3

Calculated surface energies of TaB2(0001) slabs as a function of the tantalum chemical potential. 


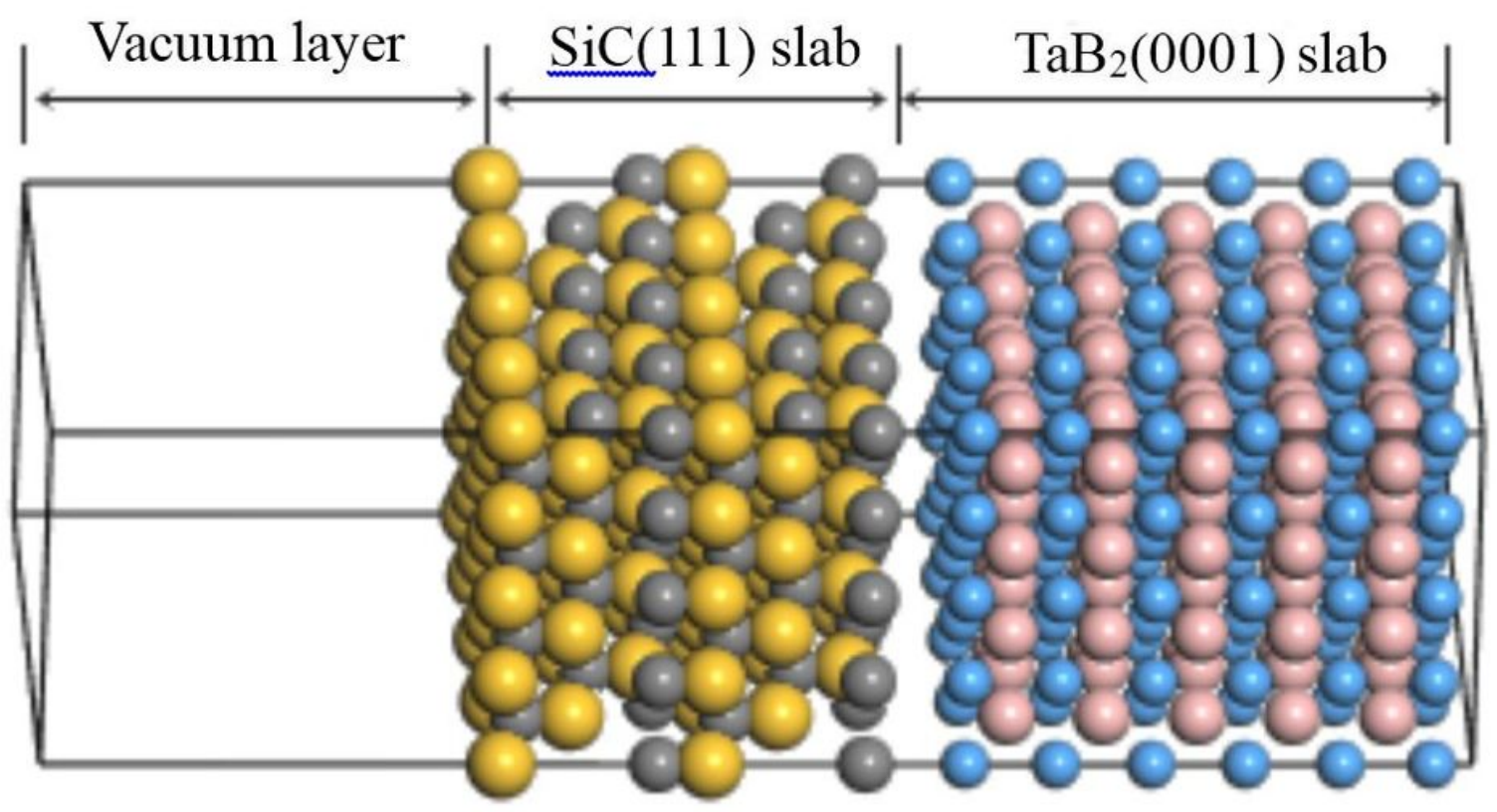

Ta

B

$\mathrm{Si}$

C

Figure 4

Schematic diagram of $\mathrm{TaB} 2(0001) / \mathrm{SiC}(111)$ interface.

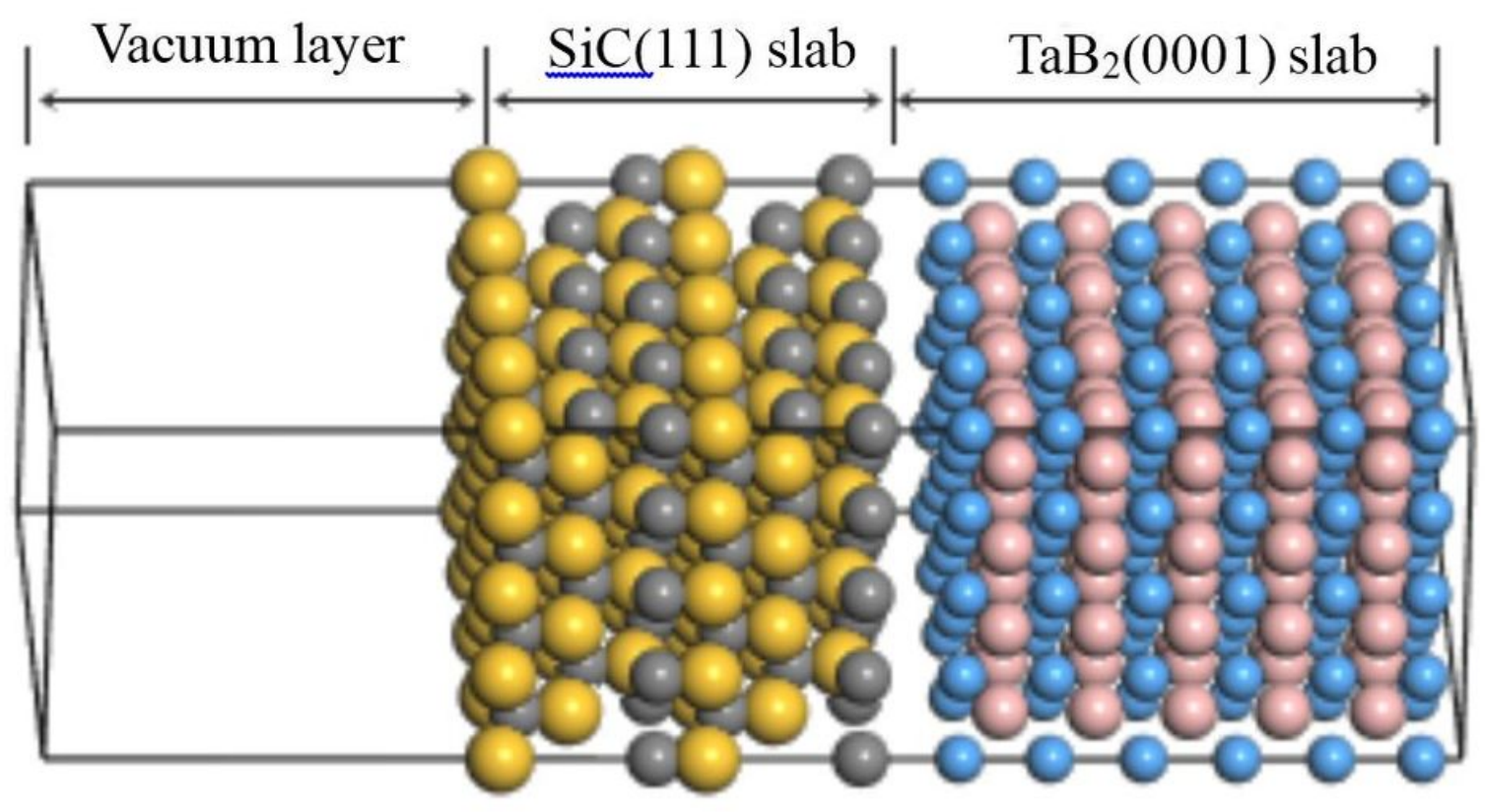

$\mathrm{Ta}$

B

$\mathrm{Si}$

C

Figure 4

Schematic diagram of $\mathrm{TaB} 2(0001) / \mathrm{SiC}(111)$ interface.

Figure 5 
Possible interfacial atomic structures of the TaB2(0001)/SiC(111) interface: (a)-(c) are the possible positions of $\mathrm{C}$ atoms on Ta-terminated TaB2(0001) surface, (d) and (e) are the possible positions of $C$ atoms on B-terminated TaB2(0001) surface.

(a)
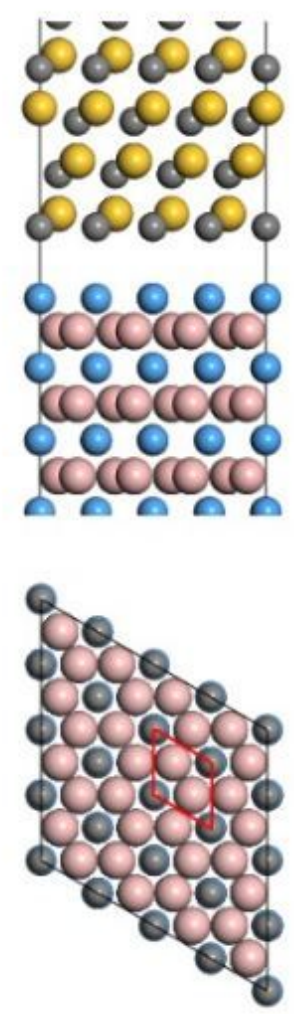

(b)
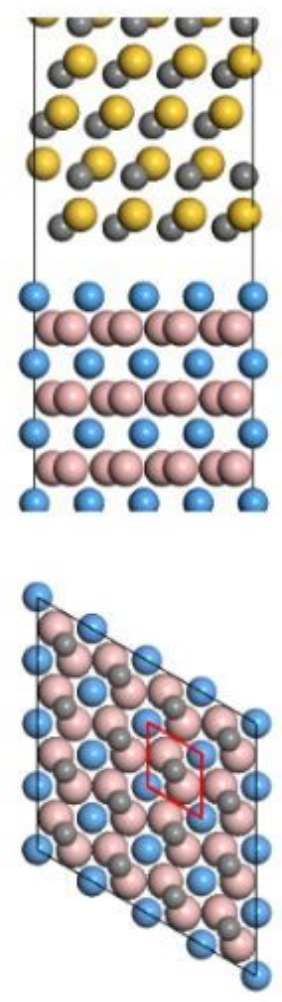

(c)
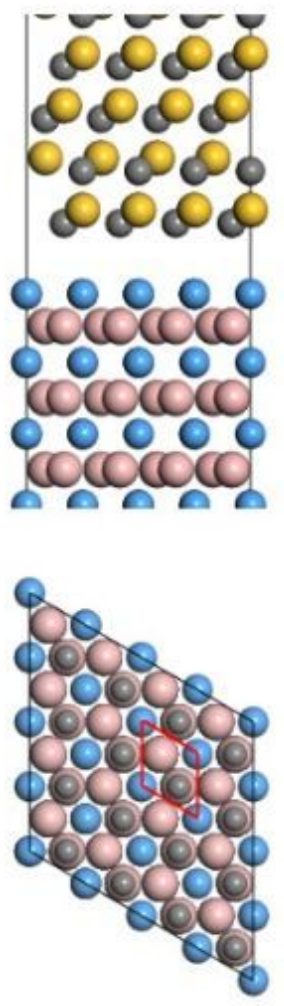

(d)
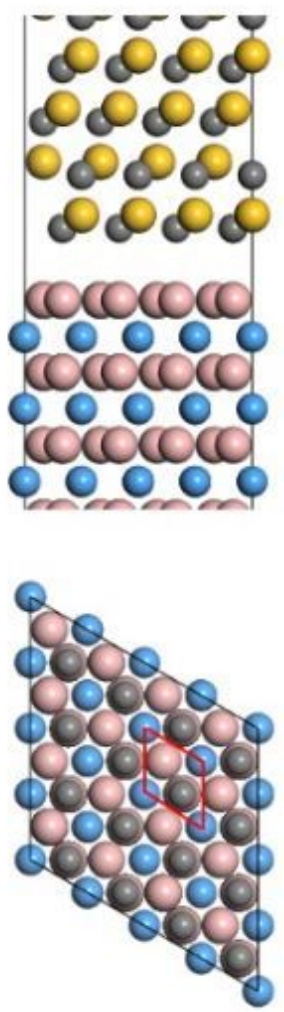

(e)

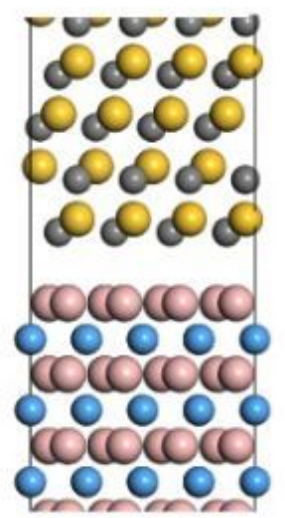

○ Ta

- B

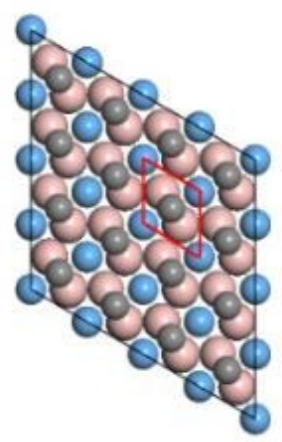

- $\mathrm{Si}$

- $\mathrm{C}$

\section{Figure 5}

Possible interfacial atomic structures of the TaB2(0001)/SiC(111) interface: (a)-(c) are the possible positions of $\mathrm{C}$ atoms on Ta-terminated TaB2(0001) surface, $(\mathrm{d})$ and $(\mathrm{e})$ are the possible positions of $\mathrm{C}$ atoms on B-terminated TaB2(0001) surface. 

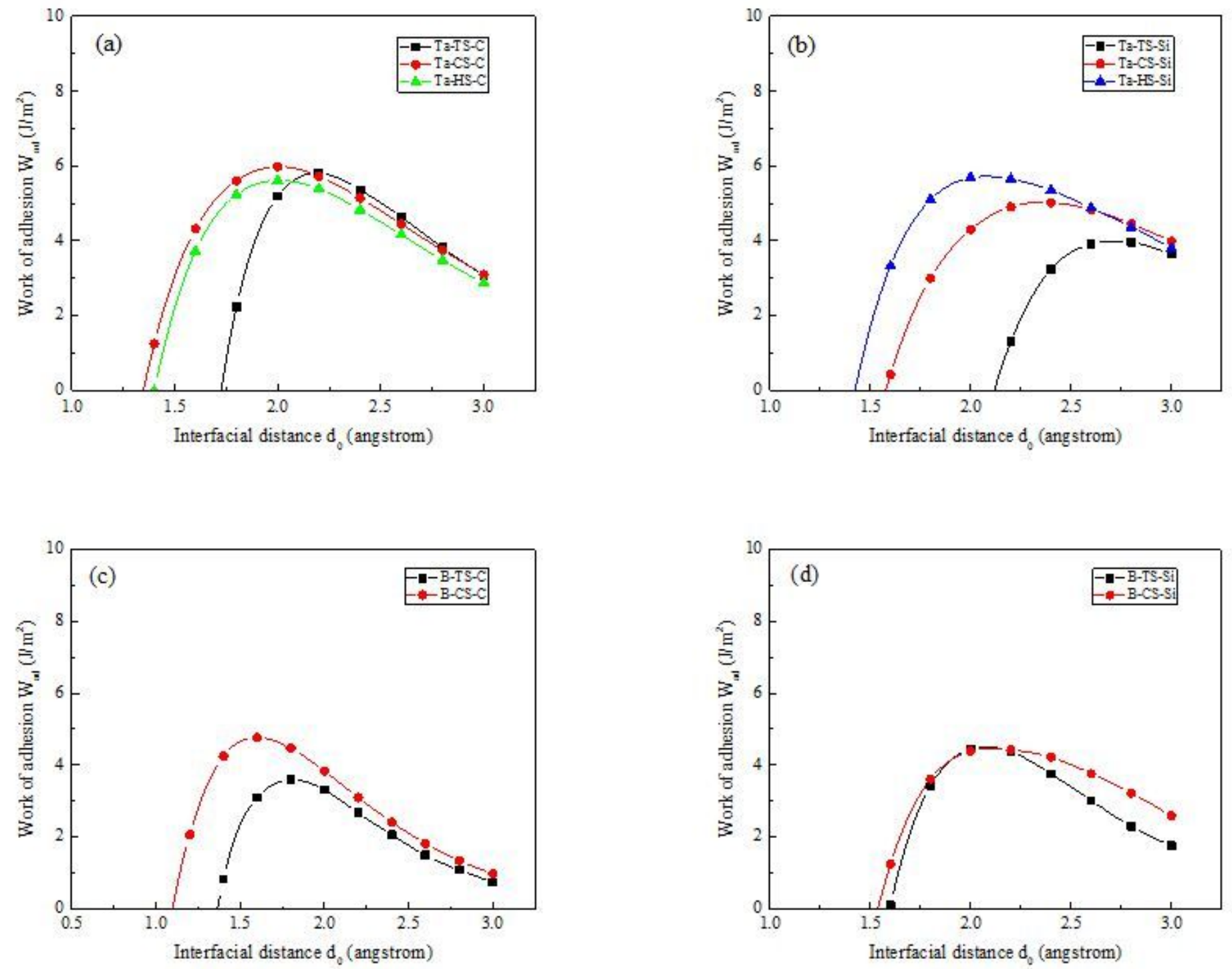

Figure 6

Interfacial work of adhesion (Wad) versus spacing distance (d0) between TaB2 and SiC slabs. 

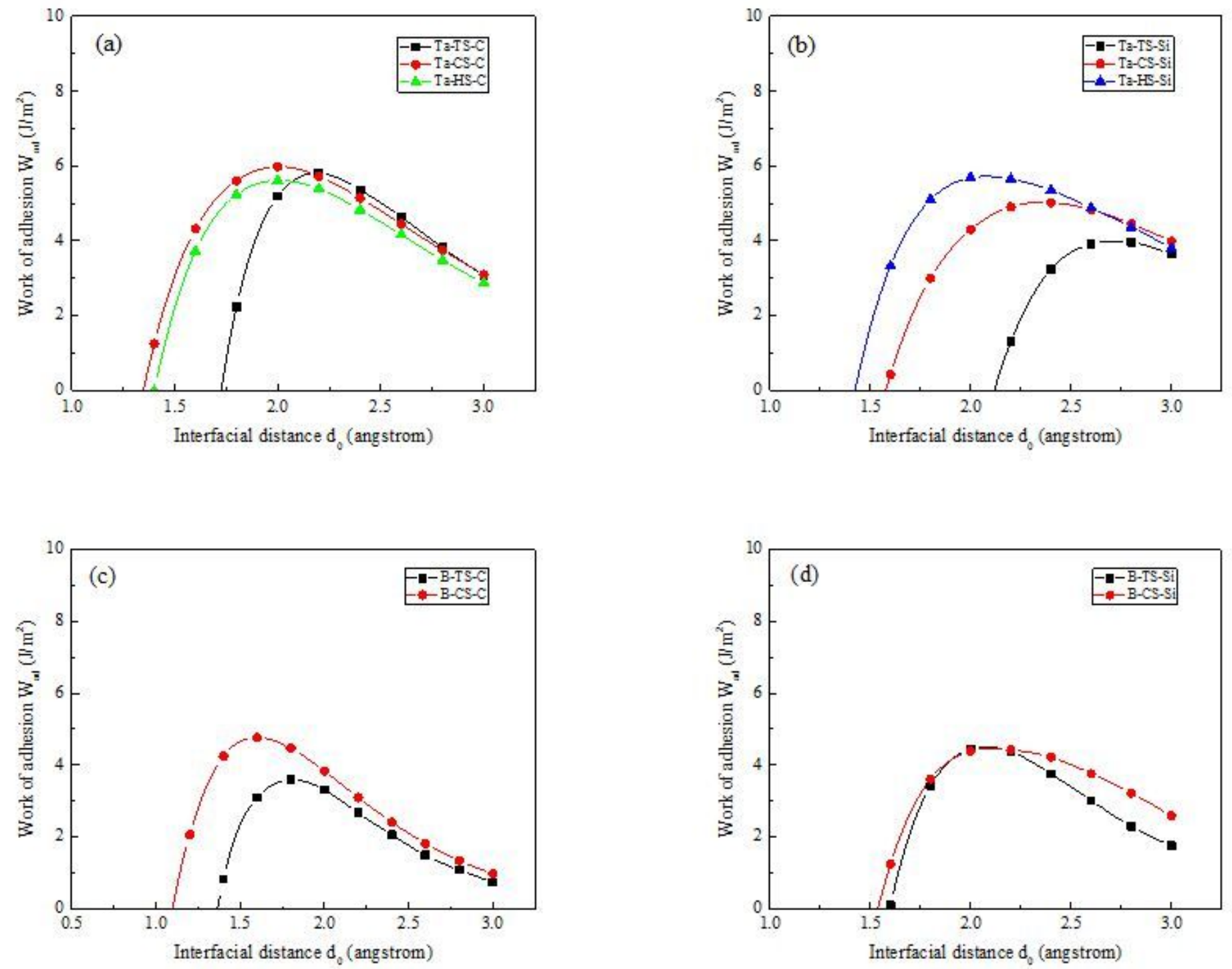

Figure 6

Interfacial work of adhesion (Wad) versus spacing distance (d0) between TaB2 and SiC slabs. 


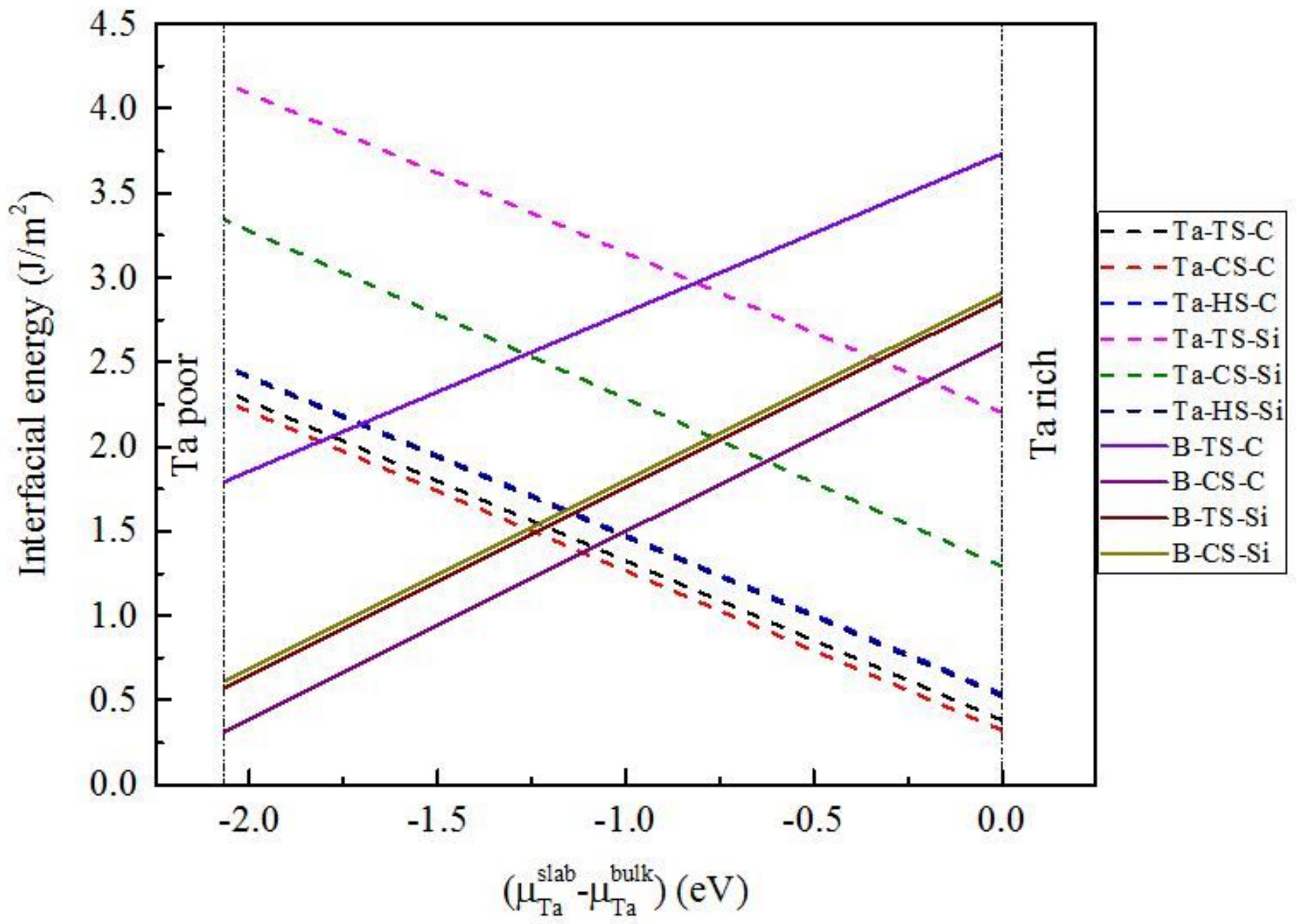

Figure 7

Interfacial energies of the ten TaB2/SiC interfaces versus chemical potential of tantalum. 


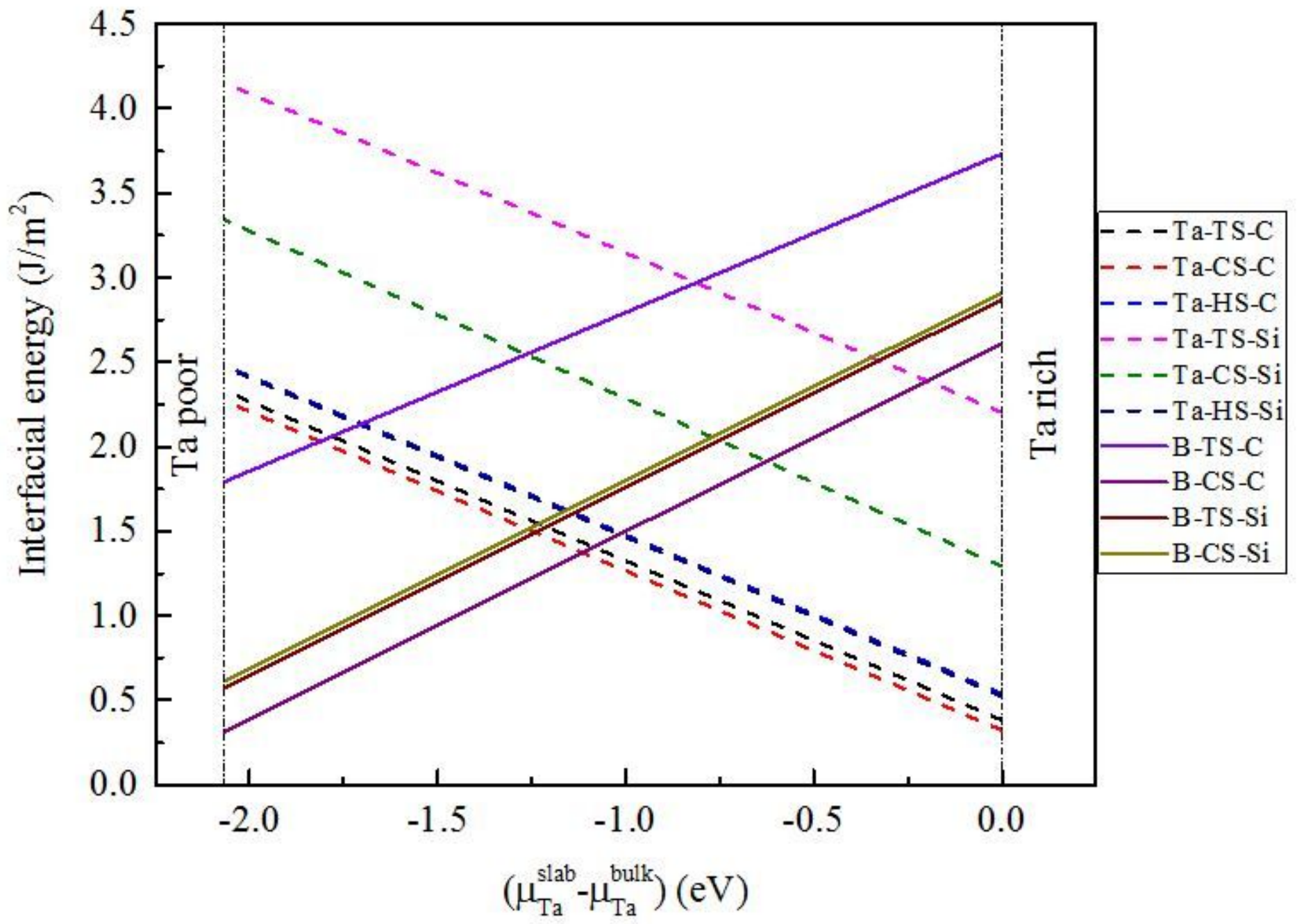

Figure 7

Interfacial energies of the ten TaB2/SiC interfaces versus chemical potential of tantalum. 
(a)

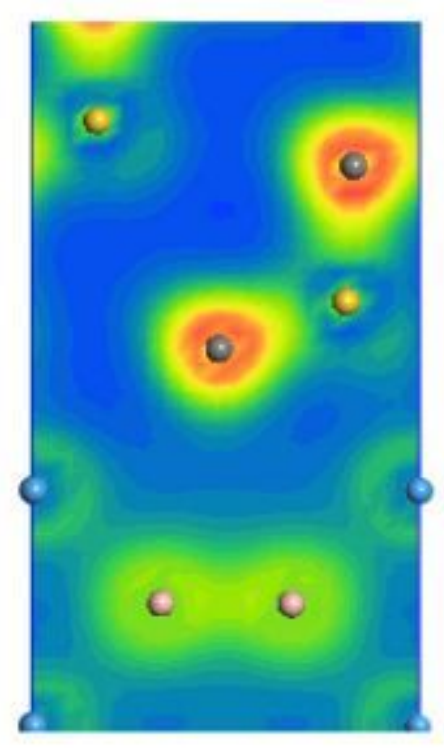

(c)

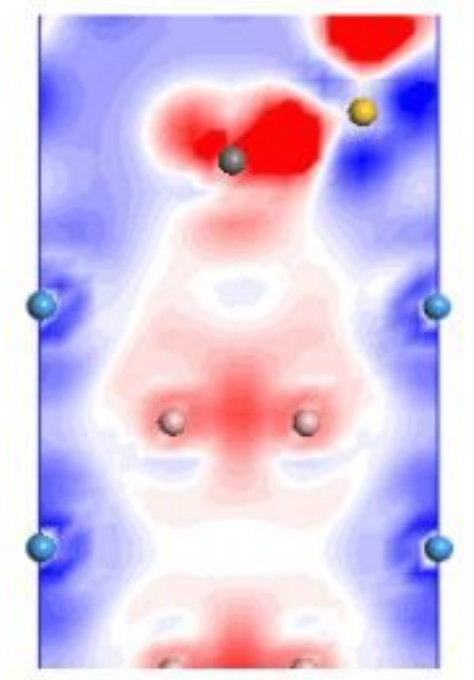

(b)

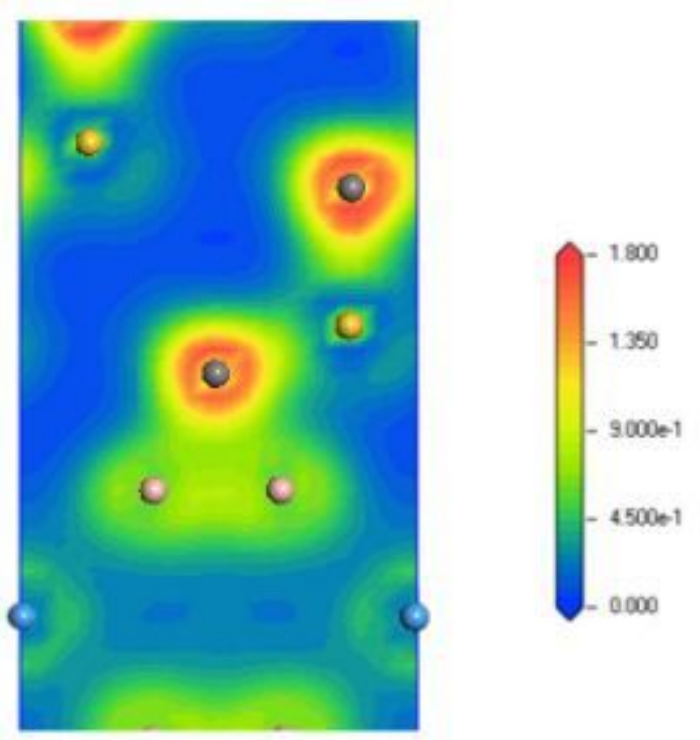

(d)
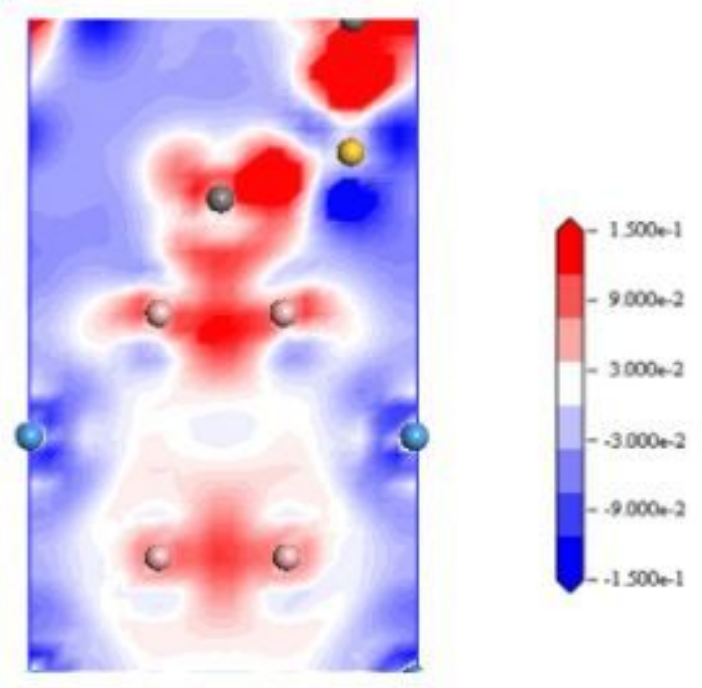

Figure 8

Charge density distribution ((a) and (b)) and charge density difference((c) and (d)) through (110) for TaCS-C and B-CS-C interfaces. 
(a)

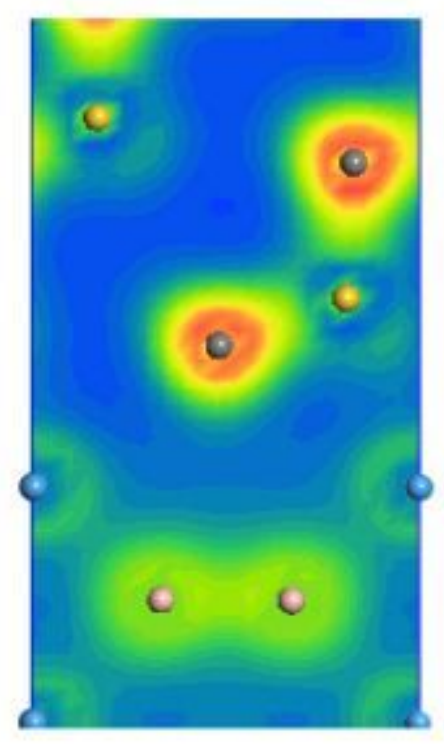

(c)

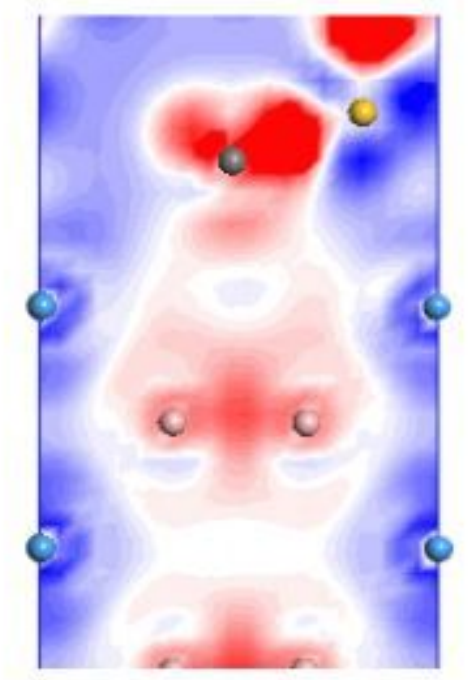

(b)

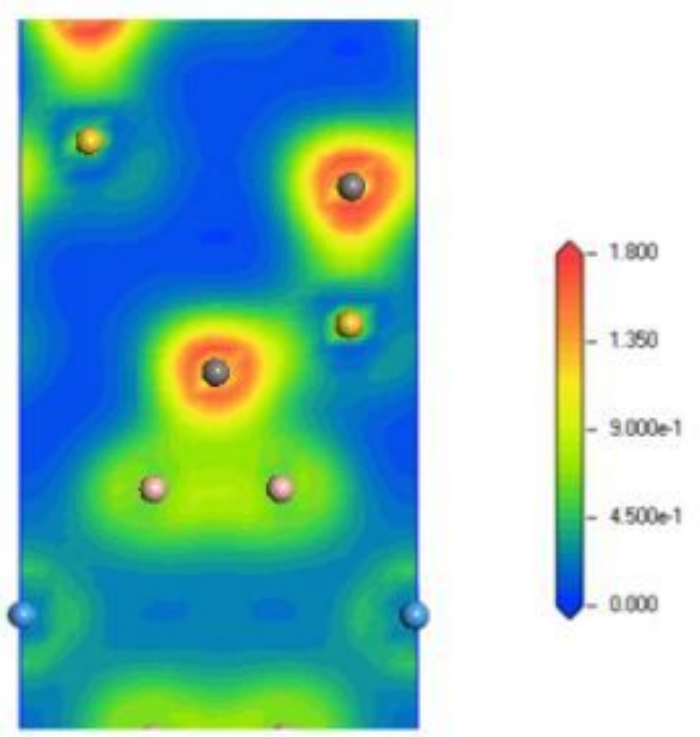

(d)
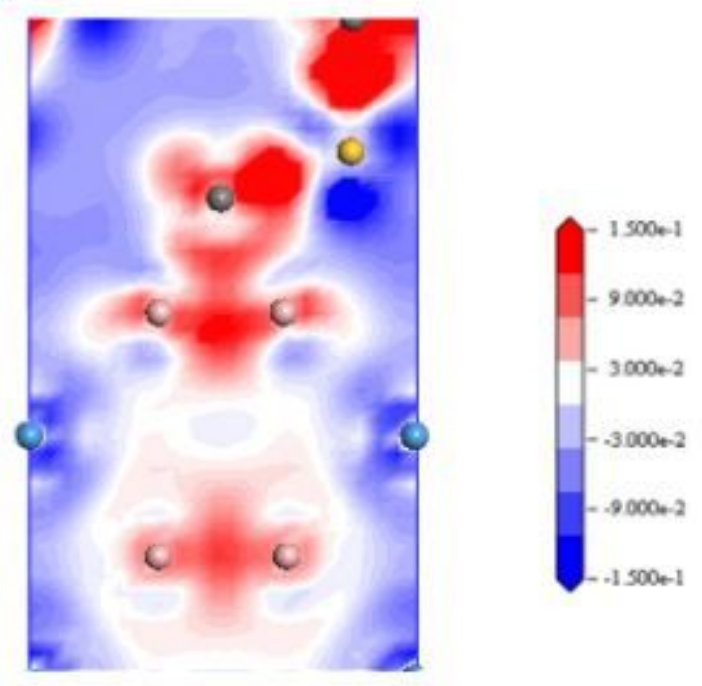

Figure 8

Charge density distribution ((a) and (b)) and charge density difference((c) and (d)) through (110) for TaCS-C and B-CS-C interfaces. 
(a)

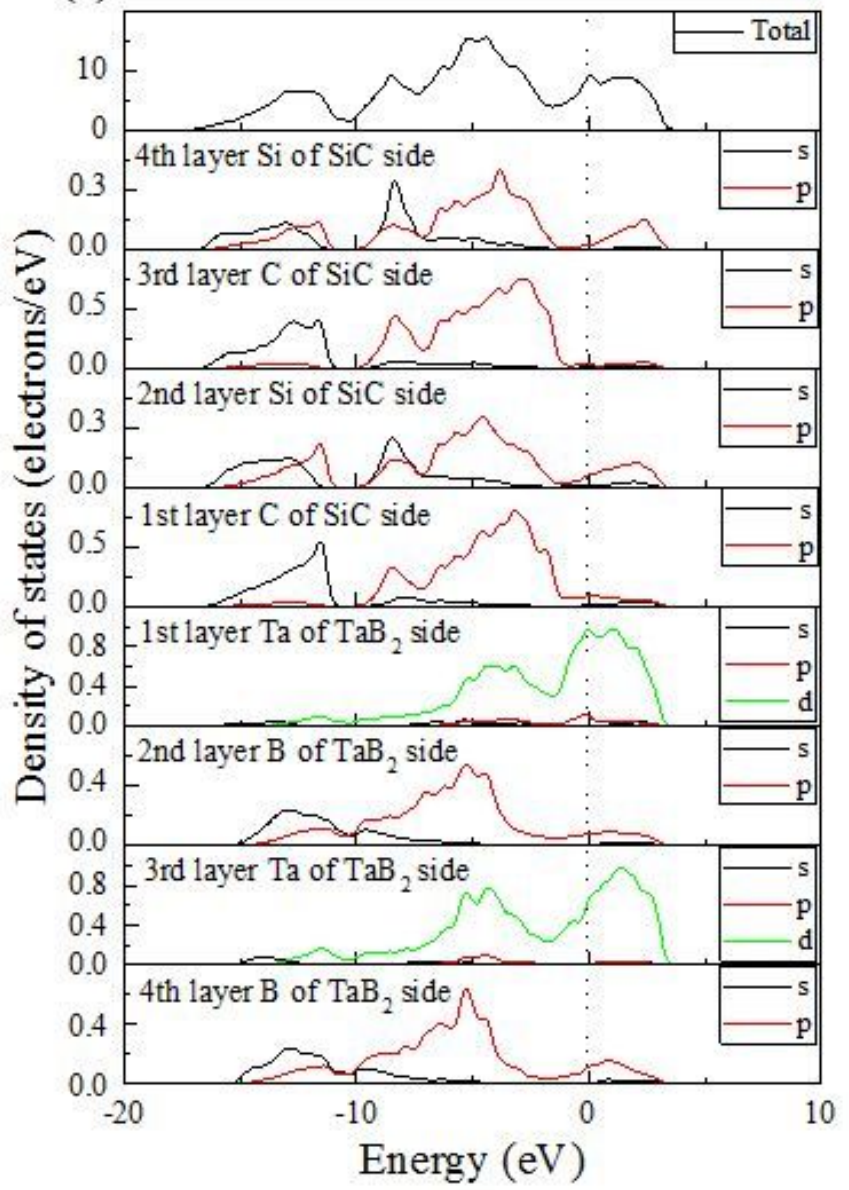

(b)

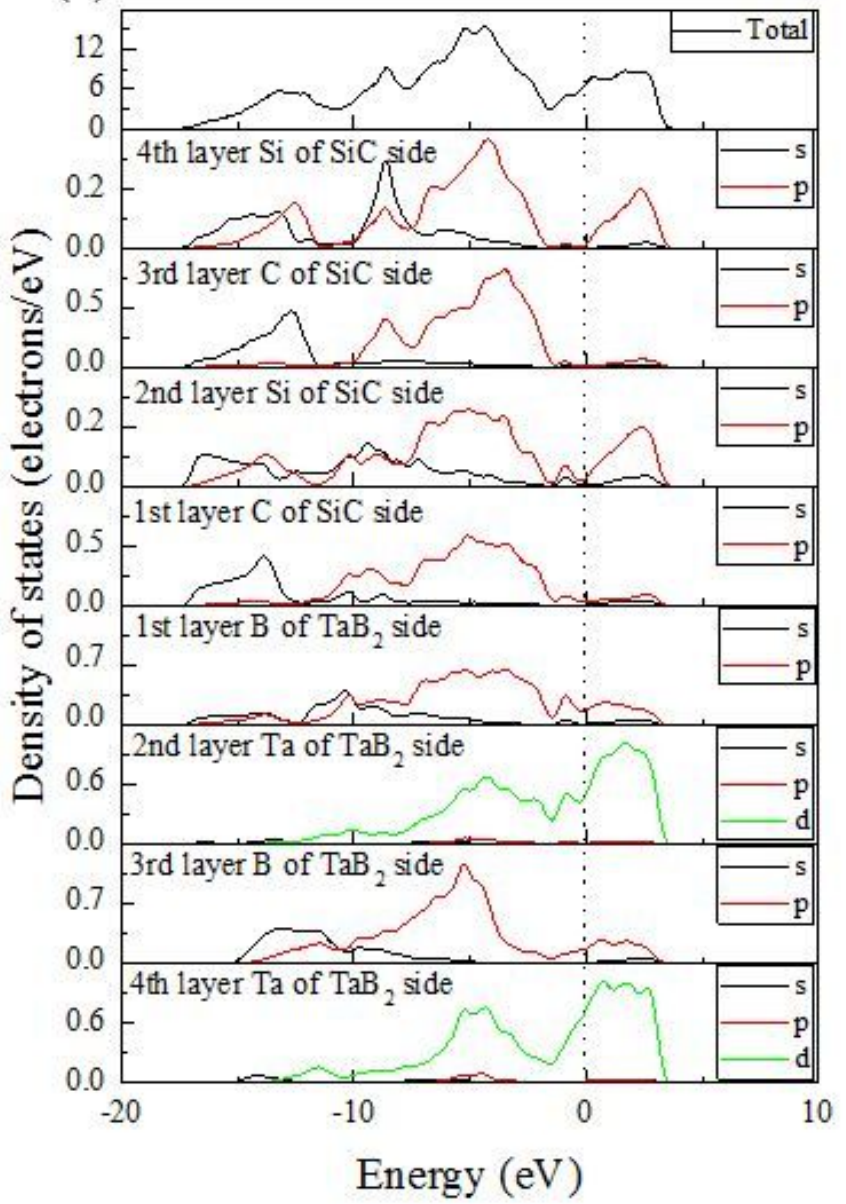

Figure 9

Layer-projected partial density of states (LPDOS) for relaxed TaB2(0001)/SiC(111) interfaces. (a) Ta-CSC; (b) B-CS-C. The dotted line indicates the location of the Fermi level. 
(a)

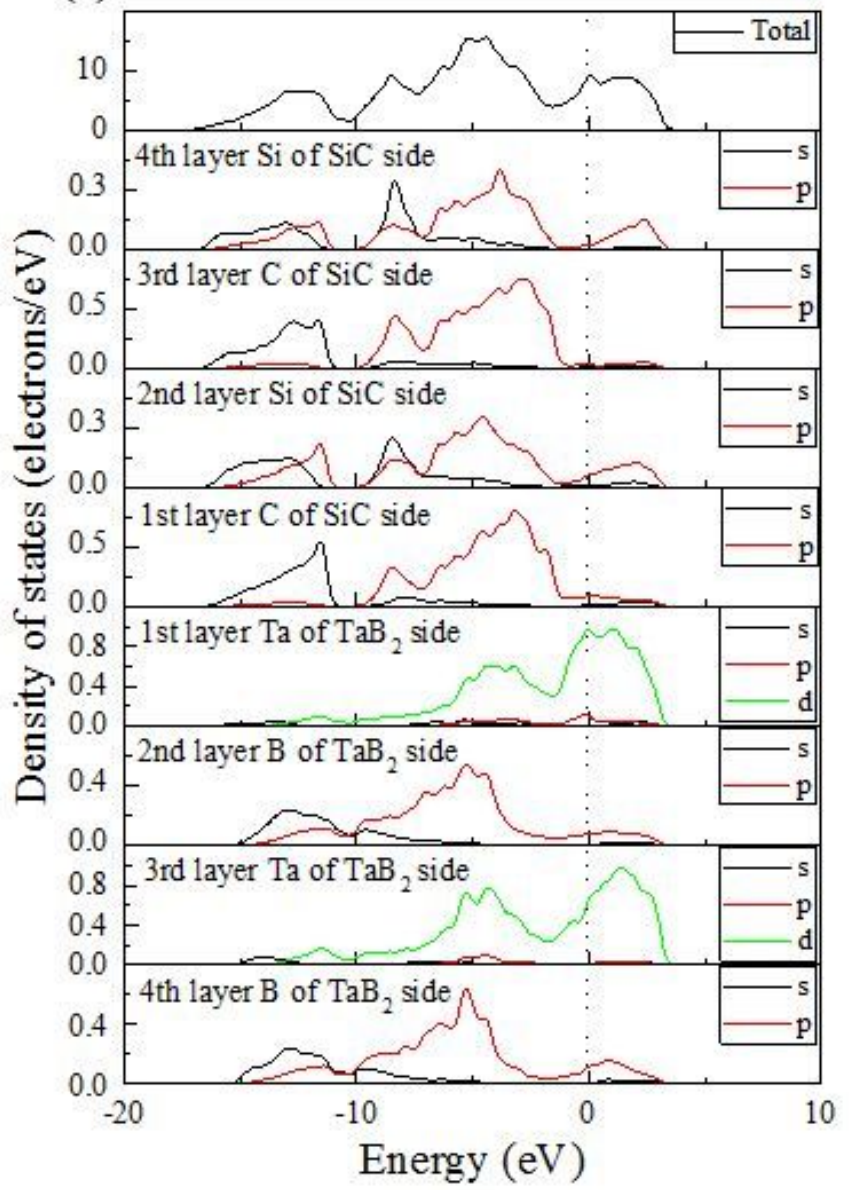

(b)

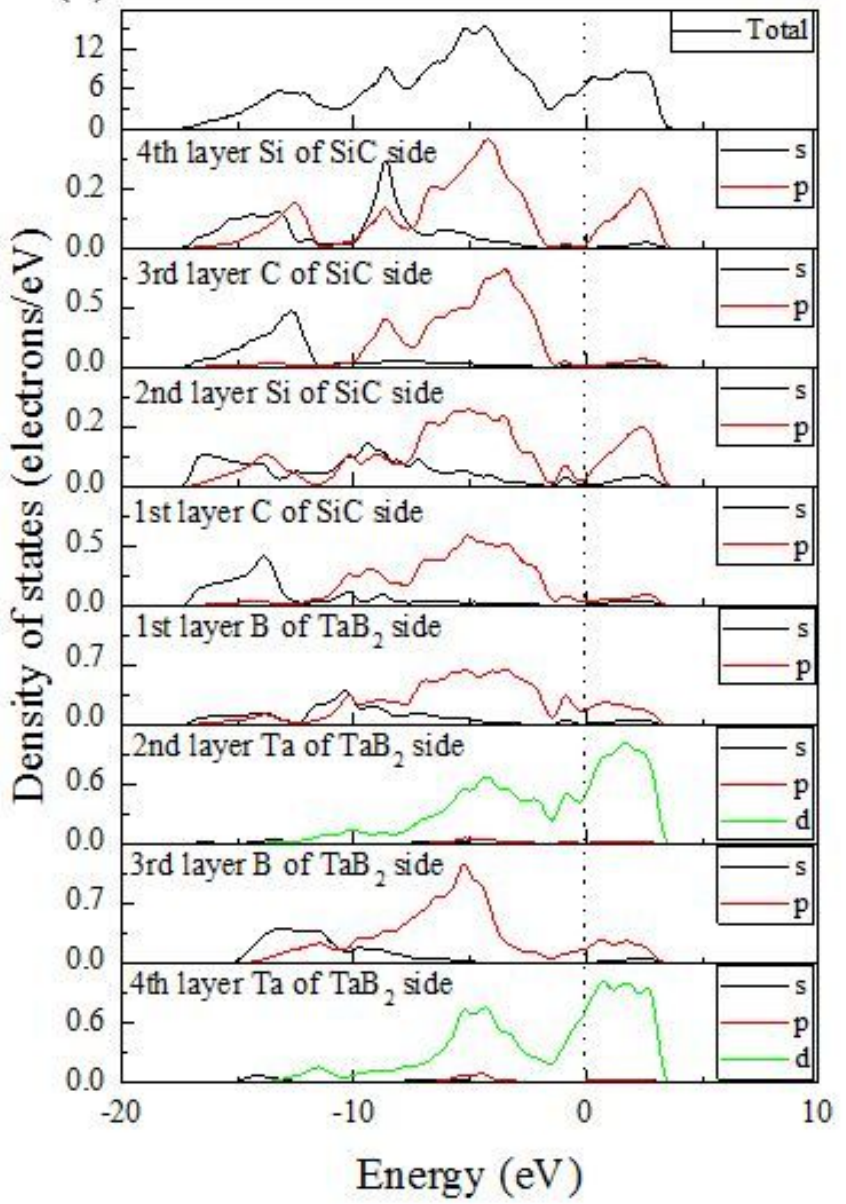

Figure 9

Layer-projected partial density of states (LPDOS) for relaxed TaB2(0001)/SiC(111) interfaces. (a) Ta-CSC; (b) B-CS-C. The dotted line indicates the location of the Fermi level. 